\title{
2,4,6-三取代嘧啶衍生物的合成及体外抗肿瘤活性研究
}

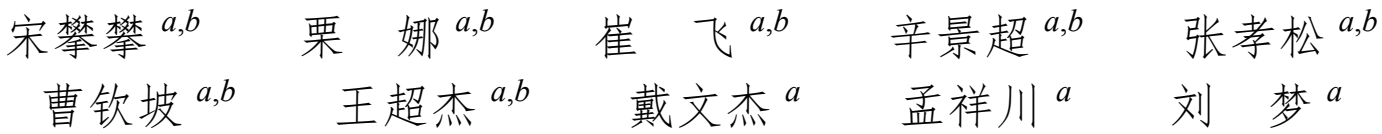

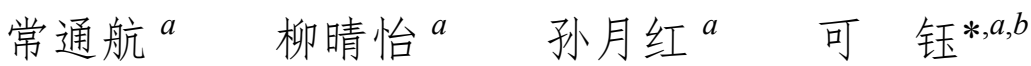 \\ 张秋荣*,a,b刘宏民*,a,b \\ ( ${ }^{a}$ 郑州大学药学院 郑州 450001) \\ ${ }^{b}$ 新药创制与药物安全性评价河南省协同创新中心 郑州 450001)
}

\begin{abstract}
摘要 为了寻找更高效、更经济的抗肿瘤药物, 以乙酰乙酸乙酯和苯甲酰乙酸乙酯为起始原料, 经环合、取代、氯代 等反应合成了一系列具有查尔酮官能团的 2,4,6-三取代嘧啶衍生物, 共 40 个化合物. 这些目标化合物的分子结构均经 过 ${ }^{1} \mathrm{H} N M R,{ }^{13} \mathrm{C}$ NMR 和 HRMS 确证, 并利用 CCK-8 方法测试它们对 MGC-803 人胃癌细胞、HepG-2 人肝癌细胞、EC-109 人食管癌细胞和 MDA-MB-231 乳腺癌细胞四种人类癌细胞系的抗肿瘤活性研究. 其中 $N-(3,4,5$-三甲氧苯乙烯基苯基 酮)-2-(苯并咪唑-2-亚甲硫基)-6-甲基嘧啶-4-胺(13u)对 MGC-803 和 MDA-MB-231 细胞株抗肿瘤活性要优于对照品 5-氟 尿嘧啶, 其 $\mathrm{IC}_{50}$ 值分别为 0.99 和 $1.77 \mu \mathrm{mol} \cdot \mathrm{L}^{-1}$.
\end{abstract}

关键词 2,4,6-三取代嘧啶; 查尔酮; 抗肿瘤

\section{Synthesis and Antitumor Activity Evaluation of 2,4,6-Trisubstituted Pyrimidine Derivatives}

\author{
Song, Panpan ${ }^{a, b}$ \\ $\mathrm{Li}, \mathrm{Na}^{a, b}$ \\ Cui, Fei ${ }^{a, b}$ \\ Xin, Jingchao ${ }^{a, b}$ \\ Zhang, Xiaosong ${ }^{a, b}$ \\ Cao, Qinpo ${ }^{a, b}$ \\ Wang, Chaojie ${ }^{a, b}$ \\ Dai, Wenjie $^{a}$ \\ Meng, Xiangchuan ${ }^{a}$ \\ Liu, Meng ${ }^{a}$ \\ Chang, Tonghang ${ }^{a}$ \\ Liu, Qingyi ${ }^{a}$ \\ Sun, Yuehong ${ }^{a}$ \\ $\mathrm{Ke}, \mathrm{Yu}^{*, a, b}$ Zhang, Qiurong ${ }^{*, a, b}$ Liu, Hongmin ${ }^{*, a, b}$ \\ ( ${ }^{a}$ School of Pharmaceutical Sciences, Zhengzhou University, Zhengzhou 450001) \\ ( ${ }^{b}$ Collaborative Innovation Center of New Drug Research and Saftry Evaluation, Zhengzhou 450001)
}

\begin{abstract}
With the aim of obtaining potential antitumor candidates with more efficiency and more economic value. 40 2,4,6-trisubstituted pyrimidine derivatives bearing chalcone moiety were synthesized via cyclization, chlorination, substitution with benzoylacetate and ethylacetoacetate as the starting materials. The structures of target products were confirmed by ${ }^{1} \mathrm{H}$ NMR, ${ }^{13} \mathrm{C}$ NMR and HRMS. 2,4,6-Trisubstituted pyrimidine derivatives bearing chalcone moiety were evaluated for anticancer activity on four human cancer cell lines including EC-109, MGC-803, HepG-2 and MDA-MB-231 by CCK-8 (cell counting Kit-8) assay. Among them, $(E)-1-(4-((2-(((1 H$-benzo[d]imidazol-2-yl)methyl)thio)-6-methylpyrimidin-4-yl)amino)phenyl)-3-(3,4,5-trimethoxyphenyl)prop-2-en-1-one (13u) were more cytotoxic against MGC-803 and MDA-MB-231 cell lines, with $\mathrm{IC}_{50}$ values of 0.99 and $1.77 \mu \mathrm{mol} \cdot \mathrm{L}^{-1}$, respectively.
\end{abstract}

Keywords 2,4,6-trisubstituted pyrimidine; chalcone; antitumor

根据由世界卫生组织下属的官方癌症机构国际癌 症研究中心(IARC)编撰最新版的《世界癌症报告》预测,
全球癌症病例将呈现迅猛增长态势，将由 2012 年的 1400 万人，逐年递增至 2025 年的 1900 万人，到 2035

\footnotetext{
* Corresponding authors. E-mail: ky@zzu.edu.cn; zqr406@sina.com; liuhm@zzu.edu.cn

Received May 8, 2017; revised May 23, 2017; published online June 7, 2017.

Project supported by the National Natural Science Foundation of China (No. 81430085) and the Research Project of Science and Technology Bureau of Zhengzhou City (No.141PQYJS554).

国家自然科学基金(No. 81430085)和郑州市科技局科研(No. 141PQYJS554)资助项目.
} 
年将达到 2400 万人. 2015 年, 美国食品药品管理局 (FDA)批准了 45 种新药, 其中 14 例(31.11\%)用于治疗癌

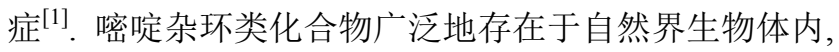
参与不同的生命活动, 是一种特别重要的生物内源物 质, 对生物体的生命活动起到重要作用. 通常具有嘧啶 环结构单元的化合物分子都具有广泛的生物和药理活 性，例如具有抗肿瘤作用的 5-氟尿嘧啶，具有抗人类免 疫缺陷病毒(HIV)的齐多夫定、具有抗病毒作用的碘苷、 具有抗菌作用的美替普林和溴莫普林、具有抗甲状腺的 丙硫氧嘧啶以及具有镇静和抗惊厥作用的苯巴比妥 等 ${ }^{[2,3]}$. 因此, 嘧啶作为药物分子设计和合成的基本砌块 已引起人们的广泛关注, 目前仍是新药分子设计、合成 与生物活性研究中的一个十分活跃的领域. 许多论文报 道, 嘧啶衍生物具有良好的抗癌活性 ${ }^{[4 ~ 7]} .1957$ 年, Duschinsky 等 ${ }^{[8]}$ 首次合成了第一代氟化嘧啶药物 5 -氟尿 嘧啶(1), 这对于肿瘤化疗治疗具有划时代的意义, 是现 代癌症化疗的重要里程碑. 1967 年, 人类首次合成了可 以口服的非肿瘤选择性药物替加氟(2). 1992 年肿瘤内激 活的靶向性口服药物氟脲苷(3)和卡培他滨(4)等都已经 成功上市(图 1$)^{[9,10]}$.

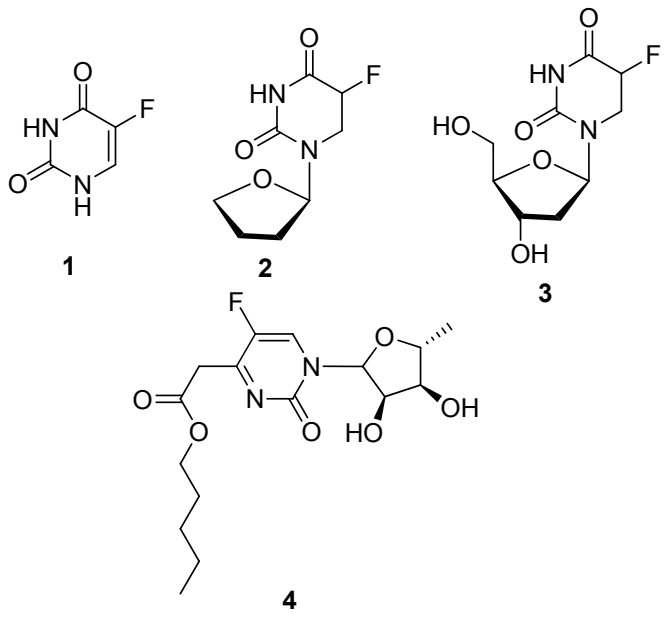

图 1 嘧啶类抗肿瘤药物的结构式

Figure 1 Structures of pyrimidines antitumor

另一方面, 查尔酮 (5) 是由烯酮连接的两个芳环构 成, 其结构的特殊性导致其具有柔性, 从而能与不同的 生物受体结合，也正是基于这个原因以及其本身的生物 活性, 无数的科研工作者将之应用于各类药物的研究 中, 得到了意想不到的成效, 尤其在抗肿瘤、抗症疾、 抗 HIV、抗菌、食用等方面得到了令人惊喜的研究成果 与进展 ${ }^{[11 ~ 14]}$. Sharma 等 $^{[15]}$ 设计合成了一系列吲哚基查 尔酮类衍生物, 并对其体外抗肿瘤活性进行评价, 活性 较好的化合物 6 和化合物 7(图 2) 对 $\mathrm{PaCa}-2$ 癌细胞系的 $\mathrm{IC}_{50}$ 值分别为 0.03 和 $0.09 \mu \mathrm{mol} / \mathrm{L}$, 且具有良好的选择
性，被认为是最具有潜力的抗癌药物. 鉴于此，我们根 据生物活性亚结构拼接原理，以嘧啶环为基本母核，设 计并合成了一系列具有查尔酮官能团的 2,4,6-三取代嘧 啶衍生物(Scheme 1), 并通过有效的合成途径将取代基 $\mathrm{R}^{1}$ 引入到 $\mathrm{C}-6$ 位, $\mathrm{R}^{2}$ 引入到 $\mathrm{C}-4$ 位, 进一步在体外评价 了其对人类癌细胞系 $\mathrm{MGC}-803$ 人胃癌细胞系、 HepG- 2 人肝癌细胞系、 EC-109人食管癌细胞系和 MDA-MB-231 乳腺癌细胞系的细胞毒活性.

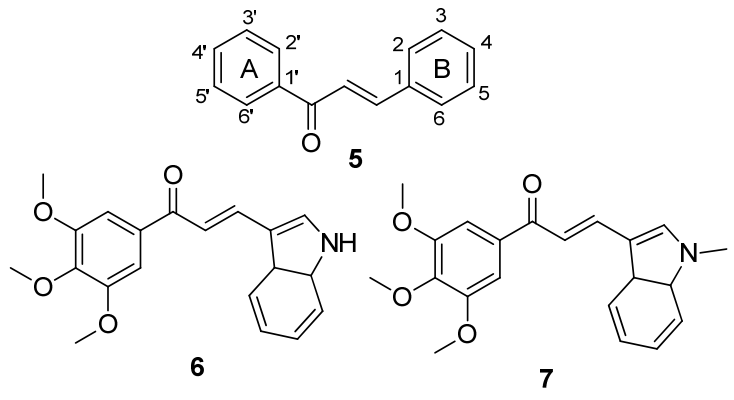

图 2 查尔酮类衍生物的结构式

Figure 2 Structures of chalcone derivatives
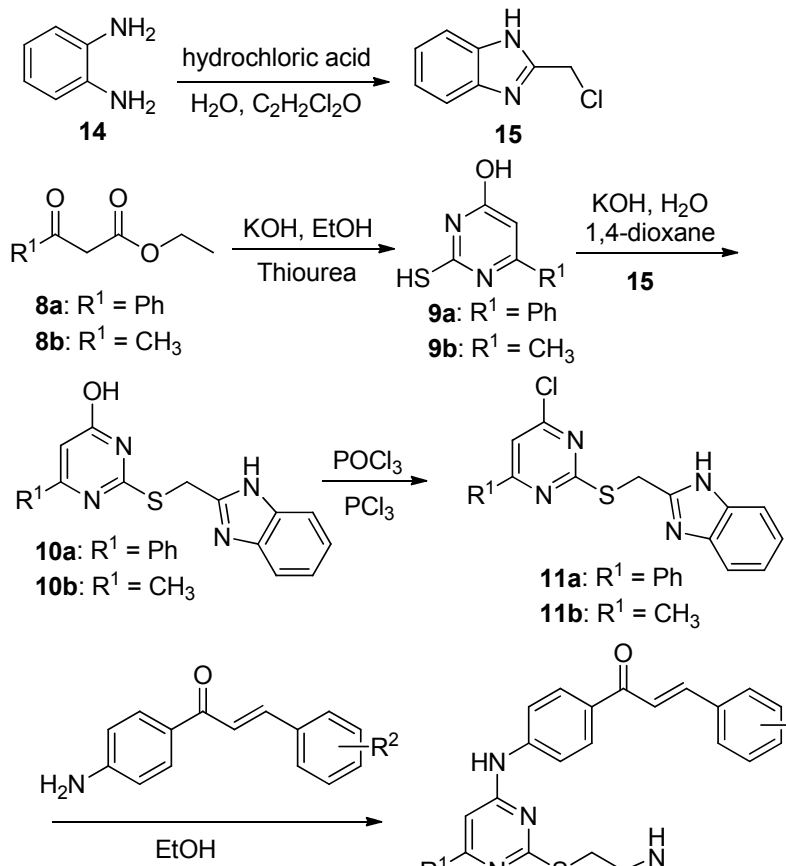<smiles></smiles>

图式 $12,4,6$-三取代嘧啶衍生物的合成路线

Scheme 1 Synthesis of 2,4,6-trisubstituted pyrimidine

\section{1 结果与讨论}

\section{1 目标化合物的合成}

目标化合物以乙酰乙酸乙酯或苯甲酰基乙酸乙酯 
为起始原料经四步反应获得. 合成路线如 Scheme 1 所 示, 我们以苯甲酰乙酸乙酯 $(8 \mathrm{a})$ 或乙酰乙酸乙酯 $(\mathbf{8 b})$ 和 硫艮为起始原料, 以无水乙醇为溶剂, 在氢氧化钾存在 条件下, $85{ }^{\circ} \mathrm{C}$ 回流 $5 \mathrm{~h}$ 发生环合反应, 以较高的产率合 成了化合物 9a 和 9b, 进而以水和 1,4-二氧六环为混合 溶剂, 将 $9 \mathbf{a}$ 和 $9 \mathbf{b}$ 与 2-氯亚甲基- $1 H$-苯并 $[d]$ 咪唑缩合得 到化合物 10a, 10b. 随后以 $\mathrm{POCl}_{3}$ 和 $\mathrm{PCl}_{3}$ 作为氯代试剂, 化合物 10a，10b 发生氯代反应，得到化合物 11a,

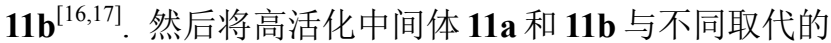
查尔酮反应，分别得到化合物 $12 \mathrm{a} \sim 12 \mathrm{o}$ 和 $13 \mathrm{a} \sim 13 \mathrm{y}$.

\section{2 化合物的生物活性分析}

采用 CCK-8 $8^{[18]}$ 比色法考察 40 个目标化合物对体外 培养的四种癌细胞 MGC-803 人胃癌细胞、HepG-2 人肝 癌细胞、EC-109人食管癌细胞和 MDA-MB-231 乳腺癌 细胞生长的抑制作用(表 1).

表 1 化合物 $12 \mathrm{a} \sim 12 \mathrm{o}$ 和 $13 \mathrm{a} \sim 13 \mathrm{y}$ 的抗肿瘤活性 $\left[\mathrm{IC}_{50} /\left(\mu \mathrm{mol} \cdot \mathrm{L}^{-1}\right)\right]^{a}$

Table 1 Antitumor activity $\left[\mathrm{IC}_{50} /\left(\mu \mathrm{mol} \cdot \mathrm{L}^{-1}\right)\right]$ of target compounds $12 \mathbf{a} \sim \mathbf{1 2 0}$ and $\mathbf{1 3 a} \sim \mathbf{1 3 y}$

\begin{tabular}{|c|c|c|c|c|c|c|}
\hline Compd. & $\mathrm{R}^{1}$ & $\mathrm{R}^{2}$ & MGC-803 & HepG-2 & EC-109 & MDA-MB-231 \\
\hline $12 \mathrm{a}$ & $\mathrm{Ph}$ & $\mathrm{H}$ & $>100$ & $33.90 \pm 1.60$ & $36.66 \pm 1.02$ & $>100$ \\
\hline $12 \mathrm{~b}$ & $\mathrm{Ph}$ & $2-\mathrm{F}$ & $>100$ & $>100$ & $>100$ & $>100$ \\
\hline $12 \mathrm{c}$ & $\mathrm{Ph}$ & $3-\mathrm{F}$ & $>100$ & $>100$ & $>100$ & $>100$ \\
\hline $12 d$ & $\mathrm{Ph}$ & $4-\mathrm{F}$ & $>100$ & $20.39 \pm 1.67$ & $55.37 \pm 1.65$ & $>100$ \\
\hline $12 \mathrm{e}$ & $\mathrm{Ph}$ & $3,4-F_{2}$ & $>100$ & $>100$ & $>100$ & $10.89 \pm 0.22$ \\
\hline $12 \mathrm{f}$ & $\mathrm{Ph}$ & $3,5-\mathrm{F}_{2}$ & $21.67 \pm 0.87$ & $27.69 \pm 0.63$ & $18.70 \pm 0.94$ & $4.11 \pm 0.34$ \\
\hline $12 \mathrm{~g}$ & $\mathrm{Ph}$ & $4-\mathrm{Cl}$ & $>100$ & $>100$ & $23.00 \pm 1.24$ & $19.90 \pm 1.13$ \\
\hline $12 \mathrm{~h}$ & $\mathrm{Ph}$ & $3,5-\mathrm{Cl}_{2}$ & $24.82 \pm 1.32$ & $9.75 \pm 1.81$ & $20.67 \pm 1.26$ & $6.45 \pm 0.65$ \\
\hline $12 \mathrm{i}$ & $\mathrm{Ph}$ & $2-\mathrm{Br}$ & $65.83 \pm 1.65$ & $76.35 \pm 1.85$ & $>100$ & $16.90 \pm 0.64$ \\
\hline $12 j$ & $\mathrm{Ph}$ & $3-\mathrm{Br}$ & $>100$ & $44.50 \pm 1.36$ & $>100$ & $>100$ \\
\hline $12 k$ & $\mathrm{Ph}$ & $4-\mathrm{Br}$ & $>100$ & $>100$ & $>100$ & $>100$ \\
\hline 121 & $\mathrm{Ph}$ & $3,5-\left(\mathrm{OCH}_{3}\right)_{2}$ & $>100$ & $>100$ & $>100$ & $>100$ \\
\hline $12 \mathrm{~m}$ & $\mathrm{Ph}$ & $4-\mathrm{HO}$ & $>100$ & $>100$ & $>100$ & $>100$ \\
\hline $12 n$ & $\mathrm{Ph}$ & $3-\mathrm{NO}_{2}$ & $>100$ & $>100$ & $>100$ & $>100$ \\
\hline 120 & $\mathrm{Ph}$ & 2-Thienyl & $>100$ & $>100$ & $>100$ & $45.86 \pm 1.54$ \\
\hline $13 a$ & $\mathrm{CH}_{3}$ & $\mathrm{H}$ & $>100$ & $>100$ & $>100$ & $>100$ \\
\hline $13 b$ & $\mathrm{CH}_{3}$ & $2-\mathrm{F}$ & $40.12 \pm 1.36$ & $31.46 \pm 1.14$ & $44.50 \pm 0.90$ & $19.99 \pm 0.65$ \\
\hline $13 \mathrm{c}$ & $\mathrm{CH}_{3}$ & $3-\mathrm{F}$ & $5.45 \pm 1.85$ & $20.67 \pm 1.38$ & $19.24 \pm 1.35$ & $8.86 \pm 1.27$ \\
\hline 13d & $\mathrm{CH}_{3}$ & $4-\mathrm{F}$ & $7.01 \pm 0.09$ & $9.92 \pm 0.36$ & $10.87 \pm 0.99$ & $9.99 \pm 0.86$ \\
\hline $13 e$ & $\mathrm{CH}_{3}$ & $3,4-F_{2}$ & $13.42 \pm 0.50$ & $13.41 \pm 0.52$ & $19.80 \pm 1.20$ & $15.00 \pm 0.71$ \\
\hline $13 f$ & $\mathrm{CH}_{3}$ & $3,5-\mathrm{F}_{2}$ & $7.15 \pm 1.43$ & $8.37 \pm 0.97$ & $14.41 \pm 0.94$ & $2.63 \pm 0.46$ \\
\hline $13 g$ & $\mathrm{CH}_{3}$ & $4-\mathrm{Cl}$ & $>100$ & $>100$ & $>100$ & $33.08 \pm 0.58$ \\
\hline $13 \mathrm{~h}$ & $\mathrm{CH}_{3}$ & $2,4-\mathrm{Cl}_{2}$ & $9.85 \pm 0.96$ & $33.33 \pm 0.33$ & $40.29 \pm 0.69$ & $8.68 \pm 0.08$ \\
\hline $13 \mathbf{i}$ & $\mathrm{CH}_{3}$ & $2,5-\mathrm{Cl}_{2}$ & $>100$ & $82.59 \pm 0.62$ & $>100$ & $31.03 \pm 0.55$ \\
\hline $13 \mathbf{j}$ & $\mathrm{CH}_{3}$ & $3,5-\mathrm{Cl}_{2}$ & $25.45 \pm 1.11$ & $>100$ & $>100$ & $>100$ \\
\hline $13 k$ & $\mathrm{CH}_{3}$ & $2-\mathrm{Br}$ & $19.14 \pm 1.11$ & $6.80 \pm 1.05$ & $25.90 \pm 1.22$ & $2.83 \pm 0.88$ \\
\hline 131 & $\mathrm{CH}_{3}$ & $3-\mathrm{Br}$ & $20.64 \pm 0.79$ & $4.89 \pm 1.21$ & $22.72 \pm 0.93$ & $10.67 \pm 0.66$ \\
\hline $13 m$ & $\mathrm{CH}_{3}$ & $4-\mathrm{Br}$ & $3.19 \pm 0.07$ & $10.05 \pm 0.06$ & $11.99 \pm 1.19$ & $12.46 \pm 0.66$ \\
\hline $13 n$ & $\mathrm{CH}_{3}$ & $2-\mathrm{F}-4-\mathrm{Br}$ & $7.61 \pm 1.24$ & $28.53 \pm 1.74$ & $17.04 \pm 1.26$ & $17.50 \pm 1.03$ \\
\hline 130 & $\mathrm{CH}_{3}$ & $4-\mathrm{F}-3-\mathrm{Br}$ & $2.90 \pm 1.36$ & $15.94 \pm 1.35$ & $23.17 \pm 1.37$ & $6.69 \pm 0.76$ \\
\hline $13 p$ & $\mathrm{CH}_{3}$ & $4-\mathrm{CH}_{3-}$ & $14.60 \pm 1.13$ & $24.19 \pm 0.80$ & $33.11 \pm 1.19$ & $17.94 \pm 0.87$ \\
\hline $13 q$ & $\mathrm{CH}_{3}$ & 4- $\mathrm{CH}\left(\mathrm{CH}_{3}\right)_{2}$ & $41.71 \pm 1.07$ & $35.54 \pm 1.23$ & $50.21 \pm 1.36$ & $48.73 \pm 0.12$ \\
\hline $13 r$ & $\mathrm{CH}_{3}$ & $4-\mathrm{C}\left(\mathrm{CH}_{3}\right)_{3}$ & $15.95 \pm 1.84$ & $36.41 \pm 1.88$ & $25.00 \pm 0.83$ & $10.91 \pm 0.08$ \\
\hline $13 \mathrm{~s}$ & $\mathrm{CH}_{3}$ & $3-\mathrm{HO}$ & $13.67 \pm 0.23$ & $18.66 \pm 0.91$ & $20.76 \pm 1.05$ & $6.77 \pm 0.84$ \\
\hline $13 t$ & $\mathrm{CH}_{3}$ & $2,3-\left(\mathrm{OCH}_{3}\right)_{2}$ & $10.62 \pm 0.95$ & $18.33 \pm 1.31$ & $58.80 \pm 1.52$ & $25.85 \pm 1.43$ \\
\hline $13 u$ & $\mathrm{CH}_{3}$ & $3,4,5-\left(\mathrm{OCH}_{3}\right)_{3}$ & $0.99 \pm 0.19$ & $21.19 \pm 0.86$ & $20.43 \pm 1.04$ & $1.77 \pm 0.27$ \\
\hline $13 v$ & $\mathrm{CH}_{3}$ & $4-\mathrm{OH}-3-\mathrm{OCH}_{3}$ & $37.88 \pm 1.40$ & $>100$ & $>100$ & $>100$ \\
\hline $13 w$ & $\mathrm{CH}_{3}$ & $3-\mathrm{NO}_{2}$ & $1.43 \pm 0.15$ & $2.20 \pm 0.38$ & $16.71 \pm 0.51$ & $3.02 \pm 0.21$ \\
\hline $13 x$ & $\mathrm{CH}_{3}$ & 2-Thienyl & $4.05 \pm 0.51$ & $5.27 \pm 0.88$ & $12.80 \pm 0.64$ & $1.05 \pm 0.14$ \\
\hline $13 y$ & $\mathrm{CH}_{3}$ & 2-Furyl & $3.49 \pm 0.69$ & $13.05 \pm 0.85$ & $23.67 \pm 1.30$ & $2.14 \pm 0.21$ \\
\hline $5-\mathrm{Fu}^{b}$ & & & $7.96 \pm 0.87$ & $10.36 \pm 0.28$ & $16.64 \pm 1.12$ & $6.58 \pm 0.38$ \\
\hline
\end{tabular}

$\bar{a}$ Antitumor activity was assayed by exposure for $72 \mathrm{~h}$ to substances and expressed as concentration required to inhibit tumor cell proliferation by $50 \%$ (IC ${ }_{50}$ ). Dates are presented as the means \pm SDs of three independent experiments. ${ }^{b}$ Positive control. 
从表 1 中的数据可以得出, 化合物 $\mathbf{1 2 a} \sim 120$ 中绝大 多数对这四种肿瘤细胞都不具有抑制活性, 遂将 $R^{1}$ 上 的苯基取代基 $(12 \mathrm{a} \sim 12 \mathrm{1})$ 改造为甲基取代基 $(13 \mathrm{a} \sim 13 \mathrm{y})$ 期待得到活性更好的化合物. 由化合物 $12 \mathrm{a} \sim 12 \mathrm{1}$ 和 $13 \mathrm{a} \sim 13 \mathrm{y}$ 的生物活性结果可以看出, 13a $\sim 13 \mathrm{y}$ 大多数化 合物对以上四种癌细胞系均有良好的抑制活性, 尤其是 对于 MGC-803, MDA-MB-231 这两个癌细胞株活性更 佳. 通过对比, 影响此类化合物生物活性的重要因素除 了查尔酮结构中 B 环上的取代基之外, 嘧啶环上 6 位取 代基对其活性影响特别大, 是决定其生物活性尤其是抗 肿瘤活性的关键因素. 当 $\mathrm{R}^{1}=\mathrm{CH}_{3}$ 时的生物活性明显优 于 $\mathrm{R}^{1}=\mathrm{Ph}$ 时的生物活性, 说明嘧啶环上 6 位小体积的给 电子取代基团更有利于化合物抗肿瘤活性的表达. 当 $\mathrm{R}^{1}=\mathrm{CH}_{3}$ 时, (1)比较化合物 13a 与其他化合物, 发现查 尔酮结构 $\mathrm{B}$ 环上没有取代基时无活性，存在取代基有利 于其活性的提高; (2)比较化合物 13p, 13q, 13r 和 13m, 13d, 发现当查尔酮 B 环的对位为吸电子基团的卤素时, 更有利于化合物抗肿瘤活性的表达; (3)分别比较 13d, $13 \mathrm{f}$ 与之对应的 $13 \mathrm{~g}, 13 \mathrm{j}$, 发现同为吸电子基团取代, $\mathrm{F}$ 原子活性要较 $\mathrm{Cl}$ 原子取代时高; (4) 比较 $13 \mathbf{h}$ 和 $\mathbf{1 3 i}, \mathbf{1 3} \mathbf{j}$, 同为查尔酮 $\mathrm{B}$ 环二氯取代, 氯原子在邻对位时活性优于 在邻位、间位或全在间位的化合物; (5)化合物 13d 和 $13 \mathrm{~m}$ 的抗肿瘤活性优于 $13 \mathrm{~b}, 13 \mathrm{c}$ 和 13k, 13l, 说明同为 吸电子卤素单取代时, 取代基处于对位时要优于邻位和 间位; (6)以化合物 13x, 13y 作为尝试, 将查尔酮的 B 环 变成 2-噻吩环或 2-呋喃环, 发现这种 5 元杂环对于抗肿 瘤活性是有利的. 由体外抗肿瘤活性评价实验结果发 现, 其中化合物 13f, 13u, 13w, 13x 对上述四种癌细胞的 抑制作用均优于或接近阳性对照 5 -氟尿嘧啶. 其中化合 物 $13 \mathrm{x}$, 对以上四种细胞的 $\mathrm{IC}_{50}$ 值分别为 $4.05,5.27$, 12.80 和 $1.05 \mu \mathrm{mol} \cdot \mathrm{L}^{-1}$, 化合物 $\mathbf{1 3} \mathbf{u}$ 对 $\mathrm{MGC}-803$ 和 MDA-MB-231 细胞株的 $\mathrm{IC}_{50}$ 值分别为 0.99 和 1.77 $\mu \mathrm{mol} \cdot \mathrm{L}^{-1}$.

\section{2 结论}

设计并制备具有查尔酮官能团的 2,4,6-三取代嘧啶 衍生物, 利用核磁共振氢谱、核磁共振碳谱和高分辨质 谱等技术证实了目标化合物的结构. 运用 CCK-8 比色 法测定了目标化合物对人类四种癌细胞 MGC- 803 人胃 癌细胞、HepG 2 人肝癌细胞、EC-109 人食管癌细胞和 MDA-MB-231 乳腺癌细胞的抗肿瘤活性, 其中化合物 13u 对 MGC-803 和 MDA-MB-231 细胞株抗肿瘤活性要 优于 5-氟尿嘧啶, 其 $\mathrm{IC}_{50}$ 值分别为 0.99 和 $1.77 \mu \mathrm{mol} \cdot$ $\mathrm{L}^{-1}$.

\section{3 实验部分}

\section{1 仪器与试剂}

${ }^{1} \mathrm{H}$ NMR 和 ${ }^{13} \mathrm{C}$ NMR 谱使用瑞典 Bruker 公司 DPX-400 型超导核磁共振仪测定, TMS 为内标; 高分辨 质谱使用美国 Waters-Micromass 公司 Q-TofMicro 高分 辨四极杆-飞行时间串联质谱仪测定. 薄层硅胶: 中国 青岛海洋化工集团公司, 柱层析硅胶: 上海五四化学试 剂厂, 氯乙酰氯: 天津市密欧化学试剂有限公司, 氢氧 化钾: 天津市化学试剂供销公司, 盐酸: 天津市永大化 学试剂有限公司, 无水乙醇: 天津市化学试剂供销公司, 1,4-二氧六环: 西陇化工有限公司. 本实验分离纯化所 用有机溶剂均为工业级, 经重新蒸馏后使用, 其他试剂 均为市售分析纯, 必要时做常规处理.

\section{2 实验方法}

\subsection{1 中间体的合成}

化合物 9a 和 9b 按照文献 $[16,17]$ 的方法制备, 化合 物 9a 和 9b 按照文献 $[16,17]$ 的方法制备, 化合物 9a 和 9b 按照文献[16，17]的方法制备，化合物 15 按照文献 $[16,17]$ 的方法制备.

\subsection{2 目标化合物 12 的合成}

将化合物 $11 \mathrm{a}(0.212 \mathrm{~g}, 0.60 \mathrm{mmol})$ 和无取代的氨基 查尔酮 $(0.110 \mathrm{~g}, 0.50 \mathrm{mmol})$ 分别溶解于 $8 \mathrm{~mL}, 2 \mathrm{~mL}$ 热的 无水乙醇中, 升温至 $90{ }^{\circ} \mathrm{C}$, 将后者缓慢滴加入加热摚 拌的前者体系中，薄层色谱法监测反应，待反应结束后 停止反应，冷却至室温后抽滤，滤饼用分析纯的无水乙 醇洗, 经真空干燥得黄色粉末状固体 $N$-(苯乙烯基苯基 酮)-2-(苯并味唑-2-亚甲硫基)-6-苯基嘧啶-4-胺(12a), $0.157 \mathrm{~g}$, 收率 $64.5 \%$. m.p. $178 \sim 179{ }^{\circ} \mathrm{C} ;{ }^{1} \mathrm{H}$ NMR $(400$ MHz, DMSO- $\left.d_{6}\right) \delta: 10.73(\mathrm{~s}, 1 \mathrm{H}), 8.18(\mathrm{~d}, J=8.8 \mathrm{~Hz}, 2 \mathrm{H})$, $8.01(\mathrm{~d}, J=15.6 \mathrm{~Hz}, 1 \mathrm{H}), 7.95 \sim 7.92(\mathrm{~m}, 2 \mathrm{H}), 7.83(\mathrm{dd}$, $J=5.1,3.5 \mathrm{~Hz}, 4 \mathrm{H}), 7.77 \sim 7.72(\mathrm{~m}, 4 \mathrm{H}), 7.48$ (dd, $J=5.5$, $3.6 \mathrm{~Hz}, 8 \mathrm{H}), 7.26(\mathrm{~s}, 1 \mathrm{H}), 5.05(\mathrm{~s}, 2 \mathrm{H}) ;{ }^{13} \mathrm{C}$ NMR $(101$ MHz, DMSO- $\left.d_{6}\right) \quad \delta: 187.20,167.74,161.57,160.63$, $151.75,144.01,143.13,135.64,134.82,131.36,131.08$, $131.03,130.79,130.44,130.05,129.94,129.16,128.90$, $128.86,128.81,128.32,128.30,126.66,126.43,126.33$, $126.03,125.69,121.99,118.71,113.72,99.93,26.34$; HR-MS (ESI) calcd for $\mathrm{C}_{33} \mathrm{H}_{26} \mathrm{~N}_{5} \mathrm{OS}[\mathrm{M}+\mathrm{H}]^{+} 540.1858$, found 540.1859 .

化合物 $12 \mathrm{~b} \sim 12 \mathrm{o}$ 的制备方法及投料比同化合物 12a.

$\mathrm{N}$-(2-氟苯乙烯基苯基酮)-2-(苯并咪唑-2-亚甲硫 基)-6-苯基嘧啶-4-胺(12b)：黄色粉末状固体， $0.151 \mathrm{~g}$, 产率 55.1\%. m.p. $190 \sim 191{ }^{\circ} \mathrm{C} ;{ }^{1} \mathrm{H}$ NMR $(400 \mathrm{MHz}$, 
DMSO- $\left.d_{6}\right) \delta: 10.78(\mathrm{~s}, 1 \mathrm{H}), 8.19(\mathrm{dd}, J=15.0,8.3 \mathrm{~Hz}$, $3 \mathrm{H}), 8.05$ (d, $J=15.7 \mathrm{~Hz}, 1 \mathrm{H}), 7.87 \sim 7.82(\mathrm{~m}, 5 \mathrm{H}), 7.74$ (dd, $J=6.2,3.2 \mathrm{~Hz}, 2 \mathrm{H}), 7.48(\mathrm{td}, J=7.3,4.1 \mathrm{~Hz}, 6 \mathrm{H})$, 7.35 (dd, $J=13.1,5.7 \mathrm{~Hz}, 2 \mathrm{H}), 7.28(\mathrm{~s}, 1 \mathrm{H}), 5.05$ (s, 2H); ${ }^{13} \mathrm{C}$ NMR (101 MHz, DMSO- $\left.d_{6}\right) \delta: 186.90,167.68$, $162.04,161.53,160.55,159.55,151.70,144.14,135.57$, $134.14,132.37,130.99,130.72,129.95,128.96,128.80$, $126.36,125.63,124.90,123.99,122.42,122.31,118.65$, 116.09, 115.88, 113.66, 99.91, 26.28; HR-MS (ESI) calcd for $\mathrm{C}_{33} \mathrm{H}_{25} \mathrm{FN}_{5} \mathrm{OS}[\mathrm{M}+\mathrm{H}]^{+}$558.1764, found 558.1762.

$N$-(3-氟苯乙烯基苯基酮)-2-(苯并咪唑-2-亚甲硫 基)-6-苯基嘧啶-4-胺(12c): 黄色粉末状固体, $0.059 \mathrm{~g}$, 产 率 30.2\%. m.p. $191 \sim 192{ }^{\circ} \mathrm{C} ;{ }^{1} \mathrm{H}$ NMR (400 MHz, DMSO- $\left.d_{6}\right) \delta: 10.62(\mathrm{~s}, 1 \mathrm{H}), 8.20(\mathrm{~d}, J=8.8 \mathrm{~Hz}, 2 \mathrm{H}), 8.06$ (d, $J=15.6 \mathrm{~Hz}, 1 \mathrm{H}), 7.90(\mathrm{~d}, J=10.2 \mathrm{~Hz}, 1 \mathrm{H}), 7.84(\mathrm{~d}, J=$ $8.4 \mathrm{~Hz}, 3 \mathrm{H}), 7.77 \sim 7.70(\mathrm{~m}, 4 \mathrm{H}), 7.56 \sim 7.43(\mathrm{~m}, 5 \mathrm{H}), 7.31$ (td, $J=8.5,2.3 \mathrm{~Hz}, 1 \mathrm{H}), 7.22(\mathrm{~s}, 1 \mathrm{H}), 5.02(\mathrm{~s}, 2 \mathrm{H}) ;{ }^{13} \mathrm{C}$ NMR $\left(101 \mathrm{MHz}\right.$, DMSO- $\left.d_{6}\right) \delta$ : 187.02, 167.75, 163.65 , $161.61,161.23,160.55,151.74,144.11,141.57,137.40$, $137.32,135.65,131.12,131.00,130.79,130.71,130.00$, $128.79,126.36,125.51,125.44,123.38,118.63,117.13$, 116.92, 114.64, 114.42, 113.70, 99.88, 26.36; HR-MS (ESI) calcd for $\mathrm{C}_{33} \mathrm{H}_{25} \mathrm{FN}_{5} \mathrm{O}_{2} \mathrm{~S}[\mathrm{M}+\mathrm{H}]^{+}$558.1764, found 558.1763.

$\mathrm{N}$-(4-氟苯乙烯基苯基酮)-2-(苯并咪唑-2-亚甲硫 基)-6-苯基嘧啶-4-胺(12d)：黄色粉末状固体，0.072 g, 产率 42.1\%. m.p. $196 \sim 197{ }^{\circ} \mathrm{C}$; ${ }^{1} \mathrm{H}$ NMR $(400 \mathrm{MHz}$, DMSO- $\left.d_{6}\right) \delta$ : $10.75(\mathrm{~s}, 1 \mathrm{H}), 8.18(\mathrm{~d}, J=8.8 \mathrm{~Hz}, 2 \mathrm{H}), 8.02$ (dd, $J=8.6,5.7 \mathrm{~Hz}, 2 \mathrm{H}), 7.96$ (s, 1H), 7.83 (dd, $J=5.1,3.5$ $\mathrm{Hz}, 4 \mathrm{H}), 7.77 \sim 7.72(\mathrm{~m}, 3 \mathrm{H}), 7.48(\mathrm{td}, J=7.3,4.3 \mathrm{~Hz}$, $5 \mathrm{H}), 7.33(\mathrm{t}, J=8.8 \mathrm{~Hz}, 2 \mathrm{H}), 7.27(\mathrm{~s}, 1 \mathrm{H}), 5.05(\mathrm{~s}, 2 \mathrm{H}) ;{ }^{13} \mathrm{C}$ NMR $\left(101 \mathrm{MHz}\right.$, DMSO- $\left.d_{6}\right) \delta: 187.13,167.76,164.55$, $162.07,161.67,160.62,151.80,143.99,141.91,135.68$, $131.53,131.37,131.19,131.10,130.80,129.95,128.88$, $126.43,125.72,121.91,118.73,116.02,115.80,113.74$, 99.93, 26.34; HR-MS (ESI) calcd for $\mathrm{C}_{33} \mathrm{H}_{25} \mathrm{FN}_{5} \mathrm{OS}[\mathrm{M}+$ $\mathrm{H}]^{+}$558.1764, found 558.1763 .

$N$-(3,4-二氟苯乙烯基苯基酮)-2-(苯并咪唑-2-亚甲 硫基)-6-苯基嘧啶-4-胺(12e): 黄色粉末状固体, $0.092 \mathrm{~g}$, 产率 50.6\%. m.p. 172 174 ${ }^{\circ} \mathrm{C} ;{ }^{1} \mathrm{H}$ NMR $(400 \mathrm{MHz}$, DMSO- $\left.d_{6}\right) \delta: 10.74(\mathrm{~s}, 1 \mathrm{H}), 8.19$ (t, $\left.J=9.7 \mathrm{~Hz}, 3 \mathrm{H}\right), 8.05$ (d, $J=15.6 \mathrm{~Hz}, 1 \mathrm{H}), 7.84(\mathrm{dd}, J=7.4,4.3 \mathrm{~Hz}, 4 \mathrm{H}), 7.73$ (t, $J=11.3 \mathrm{~Hz}, 4 \mathrm{H}), 7.59 \sim 7.45(\mathrm{~m}, 7 \mathrm{H}), 7.26(\mathrm{~s}, 1 \mathrm{H}), 5.04$ (d, $J=7.9 \mathrm{~Hz}, 2 \mathrm{H}) ;{ }^{13} \mathrm{C}$ NMR $\left(100 \mathrm{MHz}\right.$, DMSO- $\left.d_{6}\right) \delta$ : $196.57,167.79,163.87,161.65,161.42,161.29,160.52$,
$151.75,144.23,140.39,138.62,135.65,131.21,130.97$, $130.74,130.10,128.81,126.36,125.42,124.64,118.62$, $113.73,112.72,111.76,111.50,105.31,99.90,25.23$; HRMS (ESI) calcd for $\mathrm{C}_{33} \mathrm{H}_{24} \mathrm{~F}_{2} \mathrm{~N}_{5} \mathrm{OS}[\mathrm{M}+\mathrm{H}]^{+} 576.1760$, found 576.1671 .

$N$-(3,5-二氟苯乙烯基苯基酮)-2-(苯并咪夾-2-亚甲 硫基)-6-苯基嘧啶-4-胺(12f): 黄色粉末状固体, $0.158 \mathrm{~g}$, 产率 56.6\%. m.p. 222 223 ${ }^{\circ} \mathrm{C}$; ${ }^{1} \mathrm{H}$ NMR $(400 \mathrm{MHz}$, DMSO- $\left.d_{6}\right) \delta: 10.68(\mathrm{~s}, 1 \mathrm{H}), 8.22(\mathrm{~d}, J=8.8 \mathrm{~Hz}, 2 \mathrm{H}), 8.12$ $(\mathrm{d}, J=15.6 \mathrm{~Hz}, 1 \mathrm{H}), 7.88 \sim 7.82(\mathrm{~m}, 4 \mathrm{H}), 7.78 \sim 7.68(\mathrm{~m}$, $5 \mathrm{H}), 7.51 \sim 7.44(\mathrm{~m}, 5 \mathrm{H}), 7.35$ (dd, $J=10.3,8.1 \mathrm{~Hz}, 1 \mathrm{H})$, $7.24(\mathrm{~s}, 1 \mathrm{H}), 5.03(\mathrm{~s}, 2 \mathrm{H}) ;{ }^{13} \mathrm{C}$ NMR (101 MHz, DMSO- $\left.d_{6}\right)$ $\delta: 186.89,167.79,163.87,161.65,161.42,161.29,160.52$, $151.75,144.23,140.39,138.62,135.65,131.21,130.97$, $130.74,130.10,128.81,126.36,125.42,124.64,118.62$, $113.73,112.72,111.76,111.50,105.31,99.90,26.40$; HR-MS (ESI) calcd for $\mathrm{C}_{33} \mathrm{H}_{24} \mathrm{~F}_{2} \mathrm{~N}_{5} \mathrm{O}_{2} \mathrm{~S} \quad[\mathrm{M}+\mathrm{H}]^{+}$ 576.1760, found 576.1769.

$\mathrm{N}$-(4-氯苯乙烯基苯基酮)-2-(苯并咪唑-2-亚甲硫 基)-6-苯基嘧啶-4-胺(12g)：黄色粉末状固体，0.089 g, 收率 47.6\%. m.p. 209 210 ${ }^{\circ} \mathrm{C} ;{ }^{1} \mathrm{H}$ NMR $(400 \mathrm{MHz}$, DMSO- $\left.d_{6}\right) \delta: 10.72(\mathrm{~s}, 1 \mathrm{H}), 8.19(\mathrm{~d}, J=8.8 \mathrm{~Hz}, 2 \mathrm{H}), 8.01$ (dd, $J=20.4,12.1 \mathrm{~Hz}, 3 \mathrm{H}), 7.83(\mathrm{~d}, J=8.6 \mathrm{~Hz}, 4 \mathrm{H})$, $7.75 \sim 7.71(\mathrm{~m}, 3 \mathrm{H}), 7.56(\mathrm{~d}, J=8.5 \mathrm{~Hz}, 2 \mathrm{H}), 7.51 \sim 7.45$ (m, 5H), $7.26(\mathrm{~s}, 1 \mathrm{H}), 5.05(\mathrm{~s}, 2 \mathrm{H}) ;{ }^{13} \mathrm{C}$ NMR $(101 \mathrm{MHz}$, DMSO- $\left.d_{6}\right) \delta$ : $187.08,167.73,161.59,160.62,151.74$, $144.09,141.63,135.64,134.89,133.81,131.25,130.79$, $130.51,129.98,128.91,128.86,126.43,125.70,122.76$, 118.71, 113.73, 99.94, 26.33; HR-MS (ESI) calcd for $\mathrm{C}_{33} \mathrm{H}_{25} \mathrm{ClN}_{5} \mathrm{OS}[\mathrm{M}+\mathrm{H}]^{+}$574.1668, found 574.1667.

$\mathrm{N}$-(3,5-二氯苯乙烯基苯基酮)-2-(苯并咪唑-2-亚甲 硫基)-6-苯基嘧啶-4-胺(12h)：黄色粉末状固体, $0.113 \mathrm{~g}$, 产率 52.2\%. m.p. $253 \sim 254{ }^{\circ} \mathrm{C} ;{ }^{1} \mathrm{H}$ NMR $(400 \mathrm{MHz}$, DMSO- $\left.d_{6}\right) \delta: 10.72(\mathrm{~s}, 1 \mathrm{H}), 8.22(\mathrm{~d}, J=8.8 \mathrm{~Hz}, 2 \mathrm{H}), 8.15$ $(\mathrm{d}, J=15.6 \mathrm{~Hz}, 1 \mathrm{H}), 8.08(\mathrm{~d}, J=1.6 \mathrm{~Hz}, 2 \mathrm{H}), 7.88 \sim 7.82$ (m, 5H), $7.73(\mathrm{dd}, J=6.2,3.2 \mathrm{~Hz}, 2 \mathrm{H}), 7.70(\mathrm{~s}, 1 \mathrm{H})$, $7.68 \sim 7.66(\mathrm{~m}, 1 \mathrm{H}), 7.49 \sim 7.45(\mathrm{~m}, 5 \mathrm{H}), 7.25(\mathrm{~s}, 1 \mathrm{H})$, $5.03(\mathrm{~s}, 2 \mathrm{H}) ;{ }^{13} \mathrm{C}$ NMR $\left(101 \mathrm{MHz}, \mathrm{DMSO}-d_{6}\right) \delta: 191.45$, $186.89,167.83,161.72,160.58,151.84,144.31,139.96$, $138.80,138.59,135.71,134.60,133.78,131.19,131.04$, $130.80,130.20,129.22,128.86,127.56,127.17,126.43$, 125.54, 124.88, 118.68, 113.80, 99.98, 26.44; HR-MS (ESI) calcd for $\mathrm{C}_{33} \mathrm{H}_{24} \mathrm{Cl}_{2} \mathrm{~N}_{5} \mathrm{OS}[\mathrm{M}+\mathrm{H}]^{+} 608.1078$, found 608.1077.

$N$-(2-溴苯乙烯基苯基酮)-2-(苯并咪唑-2-亚甲硫 
基)-6-苯基嘧啶-4-胺(12i)：黄色粉末状固体, $0.108 \mathrm{~g}$, 产 率 46.0\%. m.p. $198 \sim 199{ }^{\circ} \mathrm{C}$; ${ }^{1} \mathrm{H}$ NMR $(400 \mathrm{MHz}$, DMSO- $\left.d_{6}\right) \delta: 10.83(\mathrm{~s}, 1 \mathrm{H}), 8.30 \sim 8.26(\mathrm{~m}, 1 \mathrm{H}), 8.20(\mathrm{~d}$, $J=8.8 \mathrm{~Hz}, 2 \mathrm{H}), 8.02$ (d, $J=1.6 \mathrm{~Hz}, 2 \mathrm{H}), 7.85$ (d, $J=8.4$ $\mathrm{Hz}, 4 \mathrm{H}), 7.79 \sim 7.71(\mathrm{~m}, 4 \mathrm{H}), 7.51 \sim 7.45(\mathrm{~m}, 6 \mathrm{H}), 7.30(\mathrm{~s}$, $1 \mathrm{H}), 5.05(\mathrm{~s}, 2 \mathrm{H}) ;{ }^{13} \mathrm{C}$ NMR (101 MHz, DMSO- $\left.d_{6}\right) \delta$ : $186.94,167.76,161.65,160.62,151.76,144.25,140.43$, $135.65,134.07,133.27,132.03,131.03,130.79,130.10$, $128.87,128.75,128.23,126.44,125.71,125.31,124.87$, 118.72, 113.73, 99.98, 26.34; HR-MS (ESI) calcd for $\mathrm{C}_{33} \mathrm{H}_{25} \mathrm{BrN}_{5} \mathrm{OS}[\mathrm{M}+\mathrm{H}]^{+}$618.0963, found 618.0966.

$N$-(3-溴苯乙烯基苯基酮)-2-(苯并咪唑-2-亚甲硫 基)-6-苯基嘧啶-4-胺(12j)：黄色粉末状固体, $0.116 \mathrm{~g}$, 产 率 50.7\%. m.p. $204 \sim 205{ }^{\circ} \mathrm{C}$; ${ }^{1} \mathrm{H}$ NMR $(400 \mathrm{MHz}$, DMSO- $\left.d_{6}\right) \delta: 10.70(\mathrm{~s}, 1 \mathrm{H}), 8.25(\mathrm{~s}, 1 \mathrm{H}), 8.21(\mathrm{~d}, J=8.7$ $\mathrm{Hz}, 2 \mathrm{H}), 8.08$ (d, $J=15.6 \mathrm{~Hz}, 1 \mathrm{H}), 7.90$ (d, $J=7.7 \mathrm{~Hz}$, 1H), 7.85 (dd, $J=7.7,3.4 \mathrm{~Hz}, 4 \mathrm{H}), 7.73$ (dd, $J=6.0,2.9$ $\mathrm{Hz}, 3 \mathrm{H}), 7.70 \sim 7.61(\mathrm{~m}, 2 \mathrm{H}), 7.49 \sim 7.44(\mathrm{~m}, 6 \mathrm{H}), 7.25(\mathrm{~s}$, 1H), $5.03(\mathrm{~s}, 2 \mathrm{H}) ;{ }^{13} \mathrm{C}$ NMR (101 MHz, DMSO-d $\left.d_{6}\right) \delta$ : $192.00,187.03,167.82,161.69,160.61,151.82,144.19$, $141.36,137.67,137.35,135.71,132.87,131.54,131.19$, $131.12,130.92,130.79,130.73,130.33,130.09,128.86$, $128.17,126.43,125.56,123.48,122.40,121.44,118.68$, 113.78, 99.95, 26.42; HR-MS (ESI) calcd for $\mathrm{C}_{33} \mathrm{H}_{25} \mathrm{BrN}_{5} \mathrm{OS}[\mathrm{M}+\mathrm{H}]^{+}$618.0963, found 618.0963.

$N$-(4-溴苯乙烯基苯基酮)-2-(苯并咪唑-2-亚甲硫 基)-6-苯基嘧啶-4-胺(12k)：黄色粉末状固体，0.153 g, 产率 60.2\%. m.p. 229 230 ${ }^{\circ} \mathrm{C} ;{ }^{1} \mathrm{H}$ NMR $(400 \mathrm{MHz}$, DMSO- $\left.d_{6}\right) \delta: 10.68(\mathrm{~s}, 1 \mathrm{H}), 8.18(\mathrm{~d}, J=8.8 \mathrm{~Hz}, 2 \mathrm{H}), 8.04$ $(\mathrm{d}, J=15.7 \mathrm{~Hz}, 1 \mathrm{H}), 7.90(\mathrm{~d}, J=8.5 \mathrm{~Hz}, 2 \mathrm{H}), 7.86 \sim 7.82$ $(\mathrm{m}, 4 \mathrm{H}), 7.77 \sim 7.67(\mathrm{~m}, 6 \mathrm{H}), 7.47(\mathrm{dd}, J=6.3,3.0 \mathrm{~Hz}$, $6 \mathrm{H}), 7.24(\mathrm{~s}, 1 \mathrm{H}), 5.03(\mathrm{~s}, 2 \mathrm{H}) ;{ }^{13} \mathrm{C} \mathrm{NMR}(101 \mathrm{MHz}$, DMSO- $\left.d_{6}\right) \delta: 187.10,167.83,161.72,160.60,151.79$, $144.08,141.74,135.73,134.13,131.85,131.27,131.14$, $130.78,130.70,129.99,128.86,126.43,125.56,123.76$, 122.80, 118.72, 113.78, 99.93, 26.43; HR-MS (ESI) calcd for $\mathrm{C}_{33} \mathrm{H}_{25} \mathrm{BrN}_{5} \mathrm{OS}[\mathrm{M}+\mathrm{H}]^{+}$618.0963, found 618.0963.

$N$-(3,5-二甲氧基苯乙烯基苯基酮)-2-(苯并咪唑-2亚甲硫基)-6-苯基嘧啶-4-胺(12I): 制备方法及投料比同 上, 得黄色粉末状固体化合物(0.100 g, 43.1\%). m.p. 207 $208{ }^{\circ} \mathrm{C} ;{ }^{1} \mathrm{H}$ NMR (400 MHz, DMSO- $d_{6}$ ) $\delta$ : 10.69 (s, $1 \mathrm{H}), 8.15$ (d, $J=8.8 \mathrm{~Hz}, 2 \mathrm{H}), 7.97$ (d, $J=9.7 \mathrm{~Hz}, 2 \mathrm{H}), 7.84$ (t, $J=7.1 \mathrm{~Hz}, 4 \mathrm{H}), 7.73(\mathrm{dd}, J=6.1,3.1 \mathrm{~Hz}, 2 \mathrm{H}), 7.68$ (dd, $J=6.5,2.9 \mathrm{~Hz}, 1 \mathrm{H}), 7.51 \sim 7.45(\mathrm{~m}, 5 \mathrm{H}), 7.25(\mathrm{~s}, 1 \mathrm{H})$, $7.21 \sim 7.16(\mathrm{~m}, 2 \mathrm{H}), 5.03(\mathrm{~d}, J=8.0 \mathrm{~Hz}, 2 \mathrm{H}), 3.84(\mathrm{~d}, J=$
$14.9 \mathrm{~Hz}, 6 \mathrm{H}) ;{ }^{13} \mathrm{C}$ NMR (101 MHz, DMSO- $\left.d_{6}\right) \delta: 187.28$, 167.79 , 161.66, 160.62, 152.75, 151.81, 148.17, 144.02, $137.10,135.70,131.39,130.95,130.77,129.90,128.85$, $128.35,126.42,125.64,124.31,122.79,119.08,118.72$, 114.82, 113.75, 99.92, 60.98, 60.26, 55.79, 26.39; HR-MS (ESI) calcd for $\mathrm{C}_{35} \mathrm{H}_{30} \mathrm{~N}_{5} \mathrm{O}_{3} \mathrm{~S}[\mathrm{M}+\mathrm{H}]^{+}$600.2069, found 600.2068 .

$N$-(4-差基苯乙烯基苯基酮)-2-(苯并咪唑-2-亚甲硫 基)-6-苯基嘧啶-4-胺(12m)：黄色粉末状固体，0.080 g, 产率收率 37.5\%. m.p. 182 $183{ }^{\circ} \mathrm{C} ;{ }^{1} \mathrm{H}$ NMR $(400 \mathrm{MHz}$, DMSO- $\left.d_{6}\right) \delta: 10.73(\mathrm{~d}, J=3.7 \mathrm{~Hz}, 1 \mathrm{H}), 8.14(\mathrm{~d}, J=8.8$ $\mathrm{Hz}, 1 \mathrm{H}), 7.92$ (d, $J=8.8 \mathrm{~Hz}, 1 \mathrm{H}), 7.87 \sim 7.72$ (m, 9H), $7.67(\mathrm{~d}, J=15.4 \mathrm{~Hz}, 1 \mathrm{H}), 7.53 \sim 7.44(\mathrm{~m}, 6 \mathrm{H}), 7.27(\mathrm{~d}, J=$ $2.3 \mathrm{~Hz}, 1 \mathrm{H}), 6.89$ (d, $J=8.6 \mathrm{~Hz}, 1 \mathrm{H}), 5.04(\mathrm{~d}, J=6.7 \mathrm{~Hz}$, $2 \mathrm{H}) ;{ }^{13} \mathrm{C}$ NMR (101 MHz, DMSO- $\left.d_{6}\right) \delta: 196.29,187.10$, $167.73,161.55,160.64,160.11,151.76,143.86,143.67$, $135.65,131.80,130.85,130.78,129.68,129.45,128.86$, $126.43,125.85,125.71,118.71,118.60,118.35,115.84$, 113.74, 99.84, 26.41, 26.29; HR-MS (ESI) calcd for $\mathrm{C}_{33} \mathrm{H}_{26} \mathrm{~N}_{5} \mathrm{O}_{2} \mathrm{~S}[\mathrm{M}+\mathrm{H}]^{+}$556.1807, found 556.1808.

$N$-(3-硝基苯乙烯基苯基酮)-2-(苯并咪唑-2-亚甲硫 基)-6-苯基嘧啶-4-胺(12n): 黄色粉末状固体，0.105 g, 产率 44.9\%. m.p. 194 $195{ }^{\circ} \mathrm{C}$; ${ }^{1} \mathrm{H}$ NMR (400 MHz, DMSO- $\left.d_{6}\right) \delta: 10.63(\mathrm{~s}, 1 \mathrm{H}), 8.81(\mathrm{~s}, 1 \mathrm{H}), 8.39$ (d, $J=7.8$ $\mathrm{Hz}, 1 \mathrm{H}), 8.30 \sim 8.27(\mathrm{~m}, 1 \mathrm{H}), 8.25 \sim 8.21(\mathrm{~m}, 3 \mathrm{H}), 8.19(\mathrm{~s}$, $1 \mathrm{H}), 7.92 \sim 7.80(\mathrm{~m}, 7 \mathrm{H}), 7.79(\mathrm{~d}, J=8.0 \mathrm{~Hz}, 1 \mathrm{H}), 7.76 \sim$ $7.72(\mathrm{~m}, 3 \mathrm{H}), 7.53 \sim 7.43(\mathrm{~m}, 7 \mathrm{H}), 7.22(\mathrm{~s}, 1 \mathrm{H}), 5.02(\mathrm{~s}$, $2 \mathrm{H}) ;{ }^{13} \mathrm{C}$ NMR (101 MHz, DMSO- $\left.d_{6}\right) \delta: 186.92,167.77$, $161.63,160.52,151.76,148.32,144.22,140.53,136.67$, $135.64,134.93,131.14,130.98,130.72,130.25,130.12$, $128.79,126.35,125.47,124.64,124.44,122.87,118.61$, 113.73, 99.89, 26.38; HR-MS (ESI) calcd for $\mathrm{C}_{33} \mathrm{H}_{25} \mathrm{~N}_{6} \mathrm{O}_{3} \mathrm{~S}$ $[\mathrm{M}+\mathrm{H}]^{+}$585.1709, found 585.1710.

$N$-(2-噻吩苯乙烯基苯基酮)-2-(苯并咪唑-2-亚甲硫 基)-6-苯基嘧啶-4-胺(120): 黄色粉末状固体, $0.091 \mathrm{~g}$, 产率 40.8\%. m.p. 204 205 ${ }^{\circ} \mathrm{C}$; ${ }^{1} \mathrm{H}$ NMR (400 MHz, DMSO-d $d_{6} \delta: 11.31(\mathrm{~s}, 1 \mathrm{H}), 10.81(\mathrm{~s}, 1 \mathrm{H}), 9.91(\mathrm{~s}, 1 \mathrm{H})$, 8.28 (d, $J=4.3 \mathrm{~Hz}, 1 \mathrm{H}), 8.03$ (t, $J=9.4 \mathrm{~Hz}, 3 \mathrm{H}), 7.93$ (t, $J=7.7 \mathrm{~Hz}, 1 \mathrm{H}), 7.63(\mathrm{dd}, J=8.1,4.6 \mathrm{~Hz}, 3 \mathrm{H}), 7.51$ (d, $J=$ $6.5 \mathrm{~Hz}, 3 \mathrm{H}), 7.14$ (t, $J=8.4 \mathrm{~Hz}, 3 \mathrm{H}), 7.09$ (s, 1H), 4.25 (s, $2 \mathrm{H}) ;{ }^{13} \mathrm{C}$ NMR (100 MHz, DMSO- $\left.d_{6}\right) \delta: 186.67,167.88$, $161.75,160.61,151.85,143.91,139.87,135.95,135.74$, 132.59 , 131.32, 131.16, 130.80, 130.19, 129.75, 128.88, $128.70,126.43,125.58,120.25,118.76,113.79$, 99.91, 26.44; HR-MS (ESI) calcd for $\mathrm{C}_{31} \mathrm{H}_{24} \mathrm{~N}_{5} \mathrm{OS}_{2}[\mathrm{M}+\mathrm{H}]^{+}$ 
546.1422, found 546.1423.

\subsection{3 目标化合物 13 的合成}

将化合物 $11 \mathrm{~b}(0.174 \mathrm{~g}, 0.60 \mathrm{mmol})$ 和无取代的氨基 查尔酮 $(0.157 \mathrm{~g}, 0.50 \mathrm{mmol})$ 分别溶解于 $8 \mathrm{~mL}, 2 \mathrm{~mL}$ 无水 乙醇中, 升温至 $90{ }^{\circ} \mathrm{C}$, 将后者缓慢滴加入加热搅拌的 前者体系中, 薄层色谱法监测反应, 待反应结束后停止 反应, 冷却至室温后抽滤, 滤饼用分析纯的无水乙醇洗 涤, 真空干燥得黄色粉末状固体 $N$-(苯乙烯基苯基 酮)-2-(苯并咪唑-2-亚甲硫基)-6-甲基嘧啶-4-胺(13a), $0.067 \mathrm{~g}$, 收率 56.6\%. m.p. 177 $178{ }^{\circ} \mathrm{C} ;{ }^{1} \mathrm{H}$ NMR (400 MHz, DMSO- $\left.d_{6}\right) \delta: 10.65(\mathrm{~s}, 1 \mathrm{H}), 8.12(\mathrm{~d}, J=8.7 \mathrm{~Hz}, 2 \mathrm{H})$, $8.04 \sim 7.91(\mathrm{~m}, 3 \mathrm{H}), 7.78 \sim 7.64(\mathrm{~m}, 5 \mathrm{H}), 7.48(\mathrm{t}, J=4.7$ $\mathrm{Hz}, 5 \mathrm{H}), 6.63$ (s, 1H), 4.97 (s, 2H), 2.27 (s, 3H); ${ }^{13} \mathrm{C} \mathrm{NMR}$ (101 MHz, DMSO- $\left.d_{6}\right) \delta: 187.79,166.24,160.33,151.09$, $143.86,143.25,135.27,132.67,131.21,131.00,130.23$, $129.41,129.37,126.16,122.43,120.21,114.21,103.02$, 26.54, 21.99; HR-MS (ESI) calcd for $\mathrm{C}_{28} \mathrm{H}_{24} \mathrm{~N}_{5} \mathrm{OS}[\mathrm{M}+$ $\mathrm{H}]^{+}$478.1701, found 478.1702 .

化合物 $13 \mathrm{~b} \sim 13 \mathrm{y}$ 的制备方法及投料比同化合物 $13 a$.

$N$-(2-氟苯乙烯基苯基酮)-2-(苯并咪唑-2-亚甲硫 基)-6-甲基嘧啶-4-胺(13b): 黄色粉末状固体, $0.120 \mathrm{~g}$, 收率 48.4\%. m.p. $178 \sim 179{ }^{\circ} \mathrm{C} ;{ }^{1} \mathrm{H}$ NMR (400 MHz, DMSO- $\left.d_{6}\right) \delta: 10.81(\mathrm{~s}, 1 \mathrm{H}), 8.21(\mathrm{t}, J=7.6 \mathrm{~Hz}, 1 \mathrm{H}), 8.06$ $(\mathrm{dd}, J=25.3,12.2 \mathrm{~Hz}, 2 \mathrm{H}), 7.87 \sim 7.79(\mathrm{~m}, 1 \mathrm{H}), 7.75 \sim$ $7.63(\mathrm{~m}, 4 \mathrm{H}), 7.61 \sim 7.43(\mathrm{~m}, 4 \mathrm{H}), 7.35(\mathrm{dd}, J=13.1,5.6$ $\mathrm{Hz}, 2 \mathrm{H}), 6.65$ (d, J=7.6 Hz, 1H), 4.98 (s, 2H), 2.27 (d, $J=$ $5.0 \mathrm{~Hz}, 3 \mathrm{H}) ;{ }^{13} \mathrm{C}$ NMR (101 MHz, DMSO- $\left.d_{6}\right) \delta: 187.54$, $166.79,164.34,162.62,160.38,160.12,151.56,143.94$, $134.85,133.11,131.98,131.22,130.36,129.55,126.21$, $125.51,124.50,122.92,122.81,119.79,116.68,116.46$, 114.23, 103.21, 26.53, 22.79; HR-MS (ESI) calcd for $\mathrm{C}_{28} \mathrm{H}_{23} \mathrm{FN}_{5} \mathrm{OS}[\mathrm{M}+\mathrm{H}]^{+}$496.1607, found 496.1607.

$N$-(3-氟苯乙烯基苯基酮)-2-(苯并咪唑-2-亚甲硫 基)-6-甲基嘧啶-4-胺(13c): 黄色粉末状固体, $0.109 \mathrm{~g}$, 收 率 55.1\%. m.p. $161 \sim 162{ }^{\circ} \mathrm{C} ;{ }^{1} \mathrm{H}$ NMR $(400 \mathrm{MHz}$, DMSO- $\left.d_{6}\right) \delta: 10.65(\mathrm{~s}, 1 \mathrm{H}), 8.12(\mathrm{~d}, J=8.7 \mathrm{~Hz}, 2 \mathrm{H})$, $8.04 \sim 7.91(\mathrm{~m}, 3 \mathrm{H}), 7.78 \sim 7.64(\mathrm{~m}, 5 \mathrm{H}), 7.48(\mathrm{t}, J=4.7$ $\mathrm{Hz}, 5 \mathrm{H}), 6.63$ (s, 1H), 4.97 (s, 2H), 2.27 (s, 3H); ${ }^{13} \mathrm{C} \mathrm{NMR}$ $\left(101 \mathrm{MHz}, \mathrm{DMSO}-d_{6}\right) \delta: 187.49,166.58,160.37,151.31$, $143.83,141.06,134.54,133.76,132.56,132.09,131.24$, $130.42,129.31,128.74,126.17,125.82,125.33,119.95$, 114.23, 103.16, 26.52, 22.48; HR-MS (ESI) calcd for $\mathrm{C}_{28} \mathrm{H}_{23} \mathrm{FN}_{5} \mathrm{OS}[\mathrm{M}+\mathrm{H}]^{+}$496.1607, found 496.1608.

$N$-(4-氟苯乙烯基苯基酮)-2-(苯并咪唑-2-亚甲硫 基)-6-甲基嘧啶-4-胺(13d)：黄色粉末状固体，0.032 g, 收率 26.9\%. m.p. 171 172 ${ }^{\circ} \mathrm{C}$; ${ }^{1} \mathrm{H}$ NMR (400 MHz, DMSO- $\left.d_{6}\right) \delta: 10.62(\mathrm{~s}, 1 \mathrm{H}), 8.12(\mathrm{~d}, J=8.8 \mathrm{~Hz}, 2 \mathrm{H})$, $8.06 \sim 7.92(\mathrm{~m}, 3 \mathrm{H}), 7.77 \sim 7.66(\mathrm{~m}, 5 \mathrm{H}), 7.48(\mathrm{dd}, J=6.1$, $3.2 \mathrm{~Hz}, 2 \mathrm{H}), 7.33(\mathrm{t}, J=8.8 \mathrm{~Hz}, 2 \mathrm{H}), 6.61(\mathrm{~d}, J=5.7 \mathrm{~Hz}$, $1 \mathrm{H}), 4.96(\mathrm{~s}, 2 \mathrm{H}), 2.26(\mathrm{~s}, 3 \mathrm{H}) ;{ }^{13} \mathrm{C} \mathrm{NMR}(101 \mathrm{MHz}$, DMSO- $\left.d_{6}\right) \delta: 187.69,166.26,165.07,162.59,160.33$, $151.09,143.28,142.60,132.62,131.98,131.95,131.76$, $131.68,131.21,130.23,126.16,122.34,120.17,116.51$, $116.29,114.89,114.21,103.03,55.86,26.53,22.03$; HR-MS (ESI) calcd for $\mathrm{C}_{28} \mathrm{H}_{23} \mathrm{FN}_{5} \mathrm{OS}[\mathrm{M}+\mathrm{H}]^{+}$496.1607, found 496.1605 .

$N$-(3,4-二氟苯乙烯基苯基酮)-2-(苯并咪唑-2-亚甲 硫基)-6-甲基嘧啶-4-胺(13e)：黄色粉末状固体, $0.194 \mathrm{~g}$, 收率 37.9\%. m.p. 165 $166{ }^{\circ} \mathrm{C} ;{ }^{1} \mathrm{H}$ NMR (400 MHz, DMSO- $\left.d_{6}\right) \delta: 10.65(\mathrm{~s}, 1 \mathrm{H}), 8.12(\mathrm{~d}, J=8.7 \mathrm{~Hz}, 2 \mathrm{H})$, $8.04 \sim 7.91(\mathrm{~m}, 3 \mathrm{H}), 7.78 \sim 7.64(\mathrm{~m}, 5 \mathrm{H}), 7.48(\mathrm{t}, J=4.7$ $\mathrm{Hz}, 5 \mathrm{H}), 6.63$ (s, 1H), 4.97 (s, 2H), 2.27 (s, 3H); ${ }^{13} \mathrm{C}$ NMR $\left(101 \mathrm{MHz}, \mathrm{DMSO}-d_{6}\right) \delta: 187.55,166.40,160.34,151.17$, $143.56,142.73,141.49,133.29,132.33,131.22,130.35$, $130.20,127.26,126.16,123.73,121.36,120.05,118.52$, $118.34,117.61,117.44,103.07,56.66,26.52,22.25$; HR-MS (ESI) calcd for $\mathrm{C}_{28} \mathrm{H}_{22} \mathrm{~F}_{2} \mathrm{~N}_{5} \mathrm{OS}[\mathrm{M}+\mathrm{H}]{ }^{+}$ 514.1513 , found 514.1511 .

$N$-(3,5-二氟苯乙烯基苯基酮)-2-(苯并咪唑-2-亚甲 硫基)-6-甲基嘧啶-4-胺(13f)：黄色粉末状固体，0.194 g, 收率 37.9\%. m.p. $182 \sim 183{ }^{\circ} \mathrm{C}$; ${ }^{1} \mathrm{H}$ NMR (400 MHz, DMSO- $\left.d_{6}\right) \delta: 10.73(\mathrm{~s}, 1 \mathrm{H}), 8.14(\mathrm{dd}, J=14.4,12.3 \mathrm{~Hz}$, $3 \mathrm{H}), 7.95 \sim 7.66(\mathrm{~m}, 7 \mathrm{H}), 7.48(\mathrm{dd}, J=6.2,3.2 \mathrm{~Hz}, 2 \mathrm{H})$, $7.41 \sim 7.30(\mathrm{~m}, 1 \mathrm{H}), 6.64(\mathrm{~s}, 1 \mathrm{H}), 4.97(\mathrm{~s}, 2 \mathrm{H}), 2.27(\mathrm{~s}$, $3 \mathrm{H}) ;{ }^{13} \mathrm{C}$ NMR (101 MHz, DMSO- $\left.d_{6}\right) \delta: 187.54,166.47$, $164.42,164.29,161.98,161.84,160.34,151.25,143.78$, $141.12,139.13,132.12,131.21,130.47,129.17,128.91$, $126.16,125.17,119.98,114.21,112.38,112.12,105.90$, 103.12, 26.52, 22.35; HR-MS (ESI) calcd for $\mathrm{C}_{28} \mathrm{H}_{22} \mathrm{~F}_{2} \mathrm{~N}_{5} \mathrm{OS}[\mathrm{M}+\mathrm{H}]^{+}$514.1513, found 514.1509.

$N$-(4-氯苯乙烯基苯基酮)-2-(苯并咪唑-2-亚甲硫 基)-6-甲基嘧啶-4-胺(13g): 黄色粉末状固体, $0.058 \mathrm{~g}$, 收率 47.2\%. m.p. 176 177 ${ }^{\circ} \mathrm{C}$; ${ }^{1} \mathrm{H}$ NMR (400 MHz, DMSO- $\left.d_{6}\right) \delta: 10.47(\mathrm{~s}, 1 \mathrm{H}), 8.12(\mathrm{~d}, J=8.8 \mathrm{~Hz}, 2 \mathrm{H}), 7.99$ $(\mathrm{dd}, J=12.0,9.9 \mathrm{~Hz}, 3 \mathrm{H}), 7.76 \sim 7.66(\mathrm{~m}, 5 \mathrm{H}), 7.56(\mathrm{~d}$, $J=8.5 \mathrm{~Hz}, 2 \mathrm{H}), 7.52 \sim 7.46(\mathrm{~m}, 2 \mathrm{H}), 6.59(\mathrm{~s}, 1 \mathrm{H}), 4.94(\mathrm{~s}$, 2H), $2.25(\mathrm{~s}, 3 \mathrm{H}) ;{ }^{13} \mathrm{C}$ NMR (101 MHz, DMSO-d $\left.d_{6}\right) \delta$ : $187.66,166.16,162.67,160.30,151.01,143.26,142.37$, $135.45,134.25,132.60,131.20,131.07,130.26,129.42$, 
$126.17,123.18,120.25,114.20,103.04,26.54,21.89$; HR-MS (ESI) calcd for $\mathrm{C}_{28} \mathrm{H}_{23} \mathrm{ClN}_{5} \mathrm{OS}[\mathrm{M}+\mathrm{H}]$ 512.1312, found 512.1313.

$N$-(2,4-二氯苯乙烯基苯基酮)-2-(苯并咪唑-2-亚甲 硫基)-6-甲基嘧啶-4-胺(13h)：黄色粉末状固体，0.120 g, 收率 54.8\%. m.p. 169 170 ${ }^{\circ} \mathrm{C}$; ${ }^{1} \mathrm{H}$ NMR $(400 \mathrm{MHz}$, DMSO- $\left.d_{6}\right) \delta: 10.74(\mathrm{~s}, 1 \mathrm{H}), 8.36(\mathrm{~d}, J=8.6 \mathrm{~Hz}, 1 \mathrm{H}), 8.11$ (dd, $J=19.9,12.1 \mathrm{~Hz}, 3 \mathrm{H}), 7.96$ (d, $J=15.5 \mathrm{~Hz}, 1 \mathrm{H})$, $7.82 \sim 7.65(\mathrm{~m}, 5 \mathrm{H}), 7.58(\mathrm{dd}, J=8.5,1.8 \mathrm{~Hz}, 1 \mathrm{H}), 7.48$ (dd, $J=6.1,3.1 \mathrm{~Hz}, 2 \mathrm{H}), 6.65$ (s, 1H), 4.97 (s, 2H), 2.27 (s, $3 \mathrm{H}) ;{ }^{13} \mathrm{C}$ NMR (101 MHz, DMSO- $\left.d_{6}\right) \delta: 187.35,166.49$, $163.55,160.34,151.25,143.82,137.05,135.96,135.56$, $132.05,131.95,131.22,130.45,130.37,129.94,128.42$, 126.18, 125.73, 119.99, 114.23, 103.16, 26.52, 22.37; HR-MS (ESI) calcd for $\mathrm{C}_{28} \mathrm{H}_{22} \mathrm{Cl}_{2} \mathrm{~N}_{5} \mathrm{OS} \quad[\mathrm{M}+\mathrm{H}]{ }^{+}$ 546.0922, found 546.0921.

$N$-(2,5-二氯苯乙烯基苯基酮)-2-(苯并咪唑-2-亚甲 硫基)-6-甲基嘧啶-4-胺(13i)：黄色粉末状固体，0.138 g, 收率 24.9\%. m.p. 174 $175{ }^{\circ} \mathrm{C} ;{ }^{1} \mathrm{H}$ NMR $(400 \mathrm{MHz}$, DMSO- $\left.d_{6}\right) \delta: 10.73(\mathrm{~s}, 1 \mathrm{H}), 7.91(\mathrm{~d}, J=8.7 \mathrm{~Hz}, 2 \mathrm{H}), 7.84$ $(\mathrm{d}, J=6.7 \mathrm{~Hz}, 2 \mathrm{H}), 7.78 \sim 7.71(\mathrm{~m}, 4 \mathrm{H}), 7.53 \sim 7.42(\mathrm{~m}$, $5 \mathrm{H}), 7.27(\mathrm{~s}, 1 \mathrm{H}), 5.03(\mathrm{~s}, 2 \mathrm{H}), 2.54(\mathrm{~s}, 3 \mathrm{H}) ;{ }^{13} \mathrm{C} \mathrm{NMR}$ $\left(100 \mathrm{MHz}, \mathrm{DMSO}-d_{6}\right) \delta: 196.8,168.2,161.8,161.1,152.2$, $144.3,136.0,131.3,129.9,129.3,126.9,126.2,119.1$, 114.2, 100.4, 26.9, 26.8; HR-MS (ESI) calcd for $\mathrm{C}_{28} \mathrm{H}_{22} \mathrm{Cl}_{2} \mathrm{~N}_{5} \mathrm{OS}[\mathrm{M}+\mathrm{H}]^{+}$554.2014, found 554.2014.

$N$-(3,5-二氯苯乙烯基苯基酮)-2-(苯并咪唑-2-亚甲 硫基)-6-甲基嘧啶-4-胺(13j): 黄色粉末状固体, $0.153 \mathrm{~g}$, 收率 56.0\%). m.p. 182 183 ${ }^{\circ} \mathrm{C} ;{ }^{1} \mathrm{H}$ NMR $(400 \mathrm{MHz}$, DMSO- $d_{6} \delta$ : $10.64(\mathrm{~s}, 1 \mathrm{H}), 8.15(\mathrm{dd}, J=12.1,10.0 \mathrm{~Hz}$, $3 \mathrm{H}), 8.09$ (d, $J=1.8 \mathrm{~Hz}, 2 \mathrm{H}), 7.80 \sim 7.64(\mathrm{~m}, 6 \mathrm{H}), 7.49$ (dd, $J=6.2,3.1 \mathrm{~Hz}, 2 \mathrm{H}), 6.62(\mathrm{~s}, 1 \mathrm{H}), 4.94$ (d, $J=5.5 \mathrm{~Hz}$, $2 \mathrm{H}), 2.25(\mathrm{~s}, 3 \mathrm{H}) ;{ }^{13} \mathrm{C}$ NMR $\left(100 \mathrm{MHz}, \mathrm{DMSO}-d_{6}\right) \delta$ : $169.3,161.8,161.2,159.5,157.2,153.6,139.6,136.8$, $136.1,131.0,129.3,127.0,123.0,122.6,116.2,115.9$, 115.8, 115.0, 98.7, 28.4; HR-MS (ESI) calcd for $\mathrm{C}_{28} \mathrm{H}_{22} \mathrm{Cl}_{2} \mathrm{~N}_{5} \mathrm{OS}[\mathrm{M}+\mathrm{H}]^{+}$546.0922, found 546.0923.

$N$-(2-溴苯乙烯基苯基酮)-2-(苯并咪唑-2-亚甲硫 基)-6-甲基嘧啶-4-胺(13k): 黄色粉末状固体, $0.070 \mathrm{~g}$, 收率 31.4\%. m.p. 170 $171{ }^{\circ} \mathrm{C} ;{ }^{1} \mathrm{H}$ NMR $(400 \mathrm{MHz}$, DMSO- $\left.d_{6}\right) \delta$ : $10.65(\mathrm{~s}, 1 \mathrm{H}), 8.12(\mathrm{~d}, J=8.7 \mathrm{~Hz}, 2 \mathrm{H})$, $8.04 \sim 7.91(\mathrm{~m}, 3 \mathrm{H}), 7.78 \sim 7.64(\mathrm{~m}, 5 \mathrm{H}), 7.48(\mathrm{t}, J=4.7$ $\mathrm{Hz}, 5 \mathrm{H}), 6.63$ (s, 1H), 4.97 (s, 2H), 2.27 (s, 3H); ${ }^{13} \mathrm{C}$ NMR $\left(101 \mathrm{MHz}, \mathrm{DMSO}-d_{6}\right) \delta: 187.66,166.63,164.21,161.79$, $160.38,151.39$, 143.79, 142.31, 137.92, 137.84, 132.21,
$131.37,131.24,130.39,126.17,126.01,123.93,119.89$, $117.73,117.51,115.27,115.06,114.23,103.11,26.51$, 22.59; HR-MS (ESI) calcd for $\mathrm{C}_{28} \mathrm{H}_{23} \mathrm{BrN}_{5} \mathrm{OS}[\mathrm{M}+\mathrm{H}]^{+}$ 556.0806, found 556.0825

$N$-(3-溴苯乙烯基苯基酮)-2-(苯并咪唑-2-亚甲硫 基)-6-甲基嘧啶-4-胺(13I): 黄色粉末状固体, $0.103 \mathrm{~g}$, 收 率 46.1\%. m.p. $169 \sim 170{ }^{\circ} \mathrm{C} ;{ }^{1} \mathrm{H}$ NMR (400 MHz, DMSO- $\left.d_{6}\right) \delta: 10.58(\mathrm{~s}, 1 \mathrm{H}), 8.24(\mathrm{~s}, 1 \mathrm{H}), 8.14(\mathrm{~d}, J=8.8$ $\mathrm{Hz}, 2 \mathrm{H}), 8.05(\mathrm{~d}, J=15.6 \mathrm{~Hz}, 1 \mathrm{H}), 7.91(\mathrm{~d}, J=7.8 \mathrm{~Hz}$, $1 \mathrm{H}), 7.79 \sim 7.58(\mathrm{~m}, 6 \mathrm{H}), 7.55 \sim 7.41(\mathrm{~m}, 3 \mathrm{H}), 6.61(\mathrm{~s}$, 1H), 4.94 (d, J=7.9 Hz, 2H), 2.26 (s, 3H); ${ }^{13} \mathrm{C}$ NMR (101 $\left.\mathrm{MHz}, \mathrm{DMSO}-d_{6}\right) \delta: 187.59,166.64,160.38,151.44$, $143.83,142.01,137.82,133.41,132.19,131.43,131.32$, $131.24,130.43,128.65,126.19,123.95,122.89,119.86$, 114.24, 103.13, 26.50, 22.62; HR-MS (ESI) calcd for $\mathrm{C}_{28} \mathrm{H}_{23} \mathrm{BrN}_{5} \mathrm{OS}[\mathrm{M}+\mathrm{H}]^{+}$556.0806, found 556.0806.

$N$-(4-溴苯乙烯基苯基酮)-2-(苯并咪唑-2-亚甲硫 基)-6-甲基嘧啶-4-胺(13m)：黄色粉末状固体，0.110 g, 收率 89.1\%. m.p. $180 \sim 181{ }^{\circ} \mathrm{C} ;{ }^{1} \mathrm{H}$ NMR $(400 \mathrm{MHz}$, DMSO- $\left.d_{6}\right) \delta: 10.60(\mathrm{~s}, 1 \mathrm{H}), 8.12(\mathrm{~d}, J=8.7 \mathrm{~Hz}, 2 \mathrm{H}), 8.02$ $(\mathrm{d}, J=15.6 \mathrm{~Hz}, 1 \mathrm{H}), 7.91(\mathrm{~d}, J=8.5 \mathrm{~Hz}, 2 \mathrm{H}), 7.79 \sim 7.65$ (m, 6H), 7.48 (dd, $J=6.1,3.2 \mathrm{~Hz}, 2 \mathrm{H}), 6.62$ (s, 1H), 4.96 $(\mathrm{s}, 3 \mathrm{H}), 2.26(\mathrm{~s}, 3 \mathrm{H}) ;{ }^{13} \mathrm{C}$ NMR (101 MHz, DMSO-d $) \delta$ : $187.66,166.18,164.03,163.57,162.90,161.25,160.31$, $151.03,143.29,142.45,134.58,132.57,132.34,131.28$, $131.20,130.27,126.17,124.33,123.23,120.23,114.21$, 103.03, 56.48, 26.54, 21.93, 19.03; HR-MS (ESI) calcd for $\mathrm{C}_{28} \mathrm{H}_{23} \mathrm{BrN}_{5} \mathrm{OS}[\mathrm{M}+\mathrm{H}]^{+}$556.0806, found 556.0803.

$N$-(2-氟-4-溴苯乙烯基苯基酮)-2-(苯并咪唑-2-亚甲 硫基)-6-甲基嘧啶-4-胺(13n)：黄色粉末状固体，0.112 g, 收率 63.2\%. m.p. 171 172 ${ }^{\circ} \mathrm{C} ;{ }^{1} \mathrm{H}$ NMR $(400 \mathrm{MHz}$, DMSO- $\left.d_{6}\right) \delta: 10.86(\mathrm{~d}, J=11.3 \mathrm{~Hz}, 1 \mathrm{H}), 8.06(\mathrm{dd}, J=$ 31.4, $12.2 \mathrm{~Hz}, 2 \mathrm{H}), 7.91(\mathrm{~d}, J=8.4 \mathrm{~Hz}, 1 \mathrm{H}), 7.82(\mathrm{~d}, J=$ $8.6 \mathrm{~Hz}, 1 \mathrm{H}), 7.74 \sim 7.65(\mathrm{~m}, 4 \mathrm{H}), 7.56(\mathrm{~d}, J=8.6 \mathrm{~Hz}, 1 \mathrm{H})$, 7.48 (ddd, $J=12.8,6.1,3.1 \mathrm{~Hz}, 2 \mathrm{H}), 6.66(\mathrm{~d}, J=6.1 \mathrm{~Hz}$, $1 \mathrm{H}), 4.97$ (d, $J=9.5 \mathrm{~Hz}, 2 \mathrm{H}), 2.28$ (s, 3H). ${ }^{13} \mathrm{C}$ NMR (100 $\left.\mathrm{MHz}, \mathrm{DMSO}-d_{6}\right) \delta: 168.4,165.8,162.2,161.2,153.7$, $144.3,136.2$, 131.3, 130.7, 130.1, 129.4, 126.9, 126.1, $123.8,119.3,115.9,114.2,100.2,60.8,27.1,14.7$; HR-MS (ESI) calcd for $\mathrm{C}_{28} \mathrm{H}_{22} \mathrm{BrFN}_{5} \mathrm{OS}[\mathrm{M}+\mathrm{H}]^{+}$574.0712, found 574.0713 .

$N$-(3-溴-4-氟苯乙烯基苯基酮)-2-(苯并咪坐-2-亚甲 硫基)-6-甲基嘧啶-4-胺(13o)：黄色粉末状固体，0.289 g, 收率 58.5\%. m.p. $179 \sim 180{ }^{\circ} \mathrm{C} ;{ }^{1} \mathrm{H}$ NMR $(400 \mathrm{MHz}$, DMSO- $\left.d_{6}\right) \delta: 11.01(\mathrm{~s}, 1 \mathrm{H}), 8.42(\mathrm{~d}, J=6.7 \mathrm{~Hz}, 1 \mathrm{H}), 8.13$ 
(d, $J=8.6 \mathrm{~Hz}, 2 \mathrm{H}), 8.02(\mathrm{dd}, J=15.5,11.0 \mathrm{~Hz}, 2 \mathrm{H})$, $7.75 \sim 7.66(\mathrm{~m}, 5 \mathrm{H}), 7.51 \sim 7.44(\mathrm{~m}, 3 \mathrm{H}), 6.70(\mathrm{~d}, J=5.0$ $\mathrm{Hz}, 1 \mathrm{H}), 5.00$ (s, 2H), 2.51 (d, $J=1.5 \mathrm{~Hz}, 3 \mathrm{H}) ;{ }^{13} \mathrm{C} \mathrm{NMR}$ $\left(100 \mathrm{MHz}, \mathrm{DMSO}-d_{6}\right) \delta: 187.03,166.02,163.20,160.41$, $159.87,157.93,150.81,143.20,140.61,133.33,131.78$, $130.73,130.61,129.89,129.28,125.68,123.18,119.45$, $117.24,117.01,113.73,108.96,108.75,102.59,26.45$, 25.99, 21.95; HR-MS (ESI) calcd for $\mathrm{C}_{28} \mathrm{H}_{22} \mathrm{BrFN}_{5} \mathrm{OS}$ $[\mathrm{M}+\mathrm{H}]^{+}$574.0712, found 574.0711.

$\mathrm{N}$-(4-甲基苯乙烯基苯基酮)-2-(苯并咪唑-2-亚甲硫 基)-6-甲基嘧啶-4-胺(13p)：黄色粉末状固体，0.125 g, 收率 50.8\%. m.p. $189 \sim 190{ }^{\circ} \mathrm{C} ;{ }^{1} \mathrm{H}$ NMR $(400 \mathrm{MHz}$, DMSO- $\left.d_{6}\right) \delta$ : $10.72(\mathrm{~s}, 1 \mathrm{H}), 8.10(\mathrm{~d}, J=8.6 \mathrm{~Hz}, 2 \mathrm{H}), 7.88$ (dd, $J=39.0,11.8 \mathrm{~Hz}, 3 \mathrm{H}), 7.78 \sim 7.62(\mathrm{~m}, 5 \mathrm{H}), 7.48(\mathrm{dd}$, $J=6.1,3.1 \mathrm{~Hz}, 2 \mathrm{H}), 7.30$ (d, $J=7.9 \mathrm{~Hz}, 2 \mathrm{H}), 6.64$ (s, $1 \mathrm{H})$, 4.97 (s, 2H), 2.37 (s, 3H), 2.27 (d, $J=5.4 \mathrm{~Hz}, 3 \mathrm{H}) ;{ }^{13} \mathrm{C}$ NMR (101 MHz, DMSO- $\left.d_{6}\right) \delta$ : 187.6, 166.00, 162.19, $160.29,150.90,143.95,142.93,141.05,132.95,132.54$, $131.20,130.12,130.03,129.41,126.15,121.34,120.37$, 114.20, 102.96, 99.99, 26.55, 21.58; HR-MS (ESI) calcd for $\mathrm{C}_{29} \mathrm{H}_{26} \mathrm{~N}_{5} \mathrm{OS}[\mathrm{M}+\mathrm{H}]^{+}$492.1858, found 492.1856.

$\mathrm{N}$-(4-异丙基苯乙烯基苯基酮)-2-(苯并咪唑-2-亚甲 硫基)-6-甲基嘧啶-4-胺(13q)：黄色粉末状固体, $0.058 \mathrm{~g}$, 收率 45.0\%. m.p. 186 187 ${ }^{\circ} \mathrm{C} ;{ }^{1} \mathrm{H}$ NMR $(400 \mathrm{MHz}$, DMSO- $\left.d_{6}\right) \delta: 10.58(\mathrm{~d}, J=14.4 \mathrm{~Hz}, 1 \mathrm{H}), 8.10(\mathrm{~d}, J=8.8$ $\mathrm{Hz}, 1 \mathrm{H}), 7.92(\mathrm{~d}, J=15.6 \mathrm{~Hz}, 1 \mathrm{H}), 7.88 \sim 7.82(\mathrm{~m}, 2 \mathrm{H})$, $7.82 \sim 7.61(\mathrm{~m}, 5 \mathrm{H}), 7.60 \sim 7.47(\mathrm{~m}, 3 \mathrm{H}), 7.36(\mathrm{~d}, J=8.2$ $\mathrm{Hz}, 1 \mathrm{H}), 6.61(\mathrm{~d}, J=6.3 \mathrm{~Hz}, 1 \mathrm{H}), 4.95(\mathrm{~d}, J=10.6 \mathrm{~Hz}$, $2 \mathrm{H}), 4.86(\mathrm{~s}, 1 \mathrm{H}), 2.26(\mathrm{~d}, J=3.2 \mathrm{~Hz}, 3 \mathrm{H}), 2.10(\mathrm{~s}, 1 \mathrm{H})$, $1.92(\mathrm{~s}, 3 \mathrm{H}), 1.24$ (d, $J=6.9 \mathrm{~Hz}, 3 \mathrm{H}) .{ }^{13} \mathrm{C}$ NMR $(101 \mathrm{MHz}$, DMSO- $\left.d_{6}\right) \delta: 187.75,166.56,160.37,151.81,151.34$, $143.88,143.46,132.98,132.55,131.23,130.19,129.52$, $127.40,126.17,121.46,119.95,114.22,103.08,33.90$, 26.53, 24.11, 22.46; HR-MS (ESI) calcd for $\mathrm{C}_{31} \mathrm{H}_{30} \mathrm{~N}_{5} \mathrm{OS}$ $[\mathrm{M}+\mathrm{H}]^{+}$520.2171, found 520.2173.

$N$-(4-叔丁基苯乙烯基苯基酮)-2-(苯并咪唑-2-亚甲 硫基)-6-甲基嘧啶-4-胺(13r)：黄色粉末状固体，0.035 g, 收率 27.3\%. m.p. 182 183 ${ }^{\circ} \mathrm{C} ;{ }^{1} \mathrm{H}$ NMR (400 MHz, DMSO- $\left.d_{6}\right) \delta: 10.64(\mathrm{~s}, 1 \mathrm{H}), 8.10(\mathrm{~d}, J=8.8 \mathrm{~Hz}, 2 \mathrm{H}), 7.89$ (dd, $J=27.1,12.0 \mathrm{~Hz}, 3 \mathrm{H}$ ), 7.70 (ddd, $J=20.5,11.0,5.5$ $\mathrm{Hz}, 5 \mathrm{H}), 7.56 \sim 7.42(\mathrm{~m}, 4 \mathrm{H}), 6.63(\mathrm{~s}, 1 \mathrm{H}), 4.97(\mathrm{~s}, 2 \mathrm{H})$, 2.27 (s, 3H), 1.32 (s, 9H); ${ }^{13} \mathrm{C}$ NMR (100 MHz, DMSO- $d_{6}$ ) $\delta: 171.2,171.1,165.6,161.6,151.2,150.8,148.6,139.1$, $134.8,132.6,129.4,128.0,120.1,113.6,113.2,36.2$; HR-MS (ESI) calcd for $\mathrm{C}_{32} \mathrm{H}_{31} \mathrm{~N}_{5} \mathrm{OS}[\mathrm{M}+\mathrm{H}]^{+}$534.2327, found 534.2330.

$N$-(3-着基苯乙烯基苯基酮)-2-(苯并咪唑-2-亚甲硫 基)-6-甲基嘧啶-4-胺(13s)：黄色粉末状固体, $0.086 \mathrm{~g}$, 收 率 56.7\%. m.p. $194 \sim 195{ }^{\circ} \mathrm{C} ;{ }^{1} \mathrm{H}$ NMR $(400 \mathrm{MHz}$, DMSO- $\left.d_{6}\right) \delta: 10.80(\mathrm{~s}, 1 \mathrm{H}), 7.86(\mathrm{~d}, J=8.6 \mathrm{~Hz}, 2 \mathrm{H}), 7.62$ (s, 1H), $7.51(\mathrm{dd}, J=6.1,3.2 \mathrm{~Hz}, 3 \mathrm{H}), 7.44$ (dd, $J=12.2$, $5.3 \mathrm{~Hz}, 3 \mathrm{H}), 7.29 \sim 7.25(\mathrm{~m}, 2 \mathrm{H}), 7.16(\mathrm{~d}, J=7.7 \mathrm{~Hz}, 1 \mathrm{H})$, $7.08(\mathrm{dd}, J=9.7,5.0 \mathrm{~Hz}, 2 \mathrm{H}), 6.73(\mathrm{dd}, J=7.9,2.0 \mathrm{~Hz}$, 1H), 6.51 (s, 1H), 4.80 (s, 2H), 2.32 (d, $J=1.7 \mathrm{~Hz}, 3 \mathrm{H})$; ${ }^{13} \mathrm{C}$ NMR (100 MHz, DMSO- $\left.d_{6}\right) \quad \delta: 187.31,165.96$, $163.02,159.88,157.80,150.83,143.62,142.92,135.99$, $132.09,130.73,129.84,129.68,129.28,125.70,121.72$, $119.67,119.57,117.73,115.31,113.74,102.55,55.98$, 26.01, 21.84, 18.52; HR-MS (ESI) calcd for $\mathrm{C}_{28} \mathrm{H}_{24} \mathrm{~N}_{5} \mathrm{O}_{2} \mathrm{~S}$ $[\mathrm{M}+\mathrm{H}]^{+}$494.1650, found 494.1650.

$N$-(2,3-二甲氧基苯乙烯基苯基酮)-2-(苯并咪唑-2亚甲硫基)-6-甲基嘧啶-4-胺(13t): 黄色粉末状固体, $0.097 \mathrm{~g}$, 收率 61.0\%. m.p. 180 181 ${ }^{\circ} \mathrm{C} ;{ }^{1} \mathrm{H}$ NMR $(400$ MHz, DMSO- $\left.d_{6}\right) \delta: 10.70(\mathrm{~s}, 1 \mathrm{H}), 8.09$ (d, $\left.J=8.8 \mathrm{~Hz}, 2 \mathrm{H}\right)$, 7.96 (q, $J=15.7 \mathrm{~Hz}, 2 \mathrm{H}), 7.70$ (ddd, $J=19.3,9.4,6.0 \mathrm{~Hz}$, $5 \mathrm{H}), 7.48(\mathrm{dd}, \quad J=6.2,3.2 \mathrm{~Hz}, 2 \mathrm{H}), 7.21 \sim 7.16(\mathrm{~m}, 2 \mathrm{H})$, $6.64(\mathrm{~s}, 1 \mathrm{H}), 4.97(\mathrm{~s}, 2 \mathrm{H}), 3.86(\mathrm{~s}, 3 \mathrm{H}), 3.82$ (s, 3H), 2.26 $(\mathrm{d}, J=5.9 \mathrm{~Hz}, 3 \mathrm{H}) ;{ }^{13} \mathrm{C}$ NMR $\left(100 \mathrm{MHz}, \mathrm{DMSO}-d_{6}\right) \delta$ : $168.1,161.9,161.1,153.7,140.7,136.1,133.3,131.3$, $131.1,130.3,130.0,129.9,129.7,129.2,126.8,125.9$, 123.6, 123.2, 119.2, 116.2, 115.9, 114.1, 99.8, 26.9; HR-MS (ESI) calcd for $\mathrm{C}_{30} \mathrm{H}_{28} \mathrm{~N}_{5} \mathrm{O}_{3} \mathrm{~S}[\mathrm{M}+\mathrm{H}]^{+}$538.1913, found 538.1915 .

$N$-(3,4,5-三甲氧苯乙烯基苯基酮)-2-(苯并咪唑-2-亚 甲硫基)-6-甲基嘧啶-4-胺(13u): 黄色粉末状固体, 0.157 g, 收率 64.4\%. m.p. 183 $184{ }^{\circ} \mathrm{C} ;{ }^{1} \mathrm{H}$ NMR (400 MHz, DMSO- $\left.d_{6}\right) \delta: 10.36(\mathrm{~s}, 1 \mathrm{H}), 8.18(\mathrm{~d}, J=8.6 \mathrm{~Hz}, 2 \mathrm{H}), 7.97$ $(\mathrm{d}, J=15.5 \mathrm{~Hz}, 1 \mathrm{H}), 7.71(\mathrm{dd}, J=10.9,8.1 \mathrm{~Hz}, 5 \mathrm{H}), 7.49$ (dd, $J=6.0,3.0 \mathrm{~Hz}, 2 \mathrm{H}), 7.28(\mathrm{~s}, 2 \mathrm{H}), 6.55(\mathrm{~s}, 1 \mathrm{H}), 4.93$ (s, 2H), $3.90(\mathrm{~s}, 6 \mathrm{H}), 3.72(\mathrm{~s}, 3 \mathrm{H}), 2.24(\mathrm{~s}, 3 \mathrm{H}) ;{ }^{13} \mathrm{C} \mathrm{NMR}$ $\left(101 \mathrm{MHz}, \mathrm{DMSO}-d_{6}\right) \delta: 187.65,166.92,164.63,160.41$, $153.61,151.58,144.23,144.02,143.80,143.46,140.11$, $132.27,131.26,130.89,130.39,126.16,121.64,119.58$, $114.21,107.01,103.17,60.61,56.73,26.54,22.98$; HR-MS (ESI) calcd for $\mathrm{C}_{31} \mathrm{H}_{30} \mathrm{~N}_{5} \mathrm{O}_{4} \mathrm{~S}[\mathrm{M}+\mathrm{H}]^{+}$568.2018, found 568.2016 .

$N$-(3-甲氧基 4-羟基二氟苯乙烯基苯基酮)-2-(苯并 咪唑-2-亚甲硫基)-6-甲基嘧啶-4-胺(13v)：黄色粉末状固 体, 0.074 g, 收率 $28.2 \%$. m.p. $179 \sim 180{ }^{\circ} \mathrm{C} ;{ }^{1} \mathrm{H}$ NMR $\left(400 \mathrm{MHz}, \mathrm{DMSO}-d_{6}\right) \delta: 10.70(\mathrm{~s}, 1 \mathrm{H}), 8.09$ (d, $J=8.8 \mathrm{~Hz}$, 
2H), 7.96 (q, $J=15.7 \mathrm{~Hz}, 2 \mathrm{H}), 7.70$ (ddd, $J=19.3,9.4,6.0$ $\mathrm{Hz}, 5 \mathrm{H}), 7.48(\mathrm{dd}, J=6.2,3.2 \mathrm{~Hz}, 2 \mathrm{H}), 7.21 \sim 7.16(\mathrm{~m}$, 2H), 6.64 (s, 1H), 4.97 (s, 2H), $3.86(\mathrm{~s}, 3 \mathrm{H}), 3.82$ (s, 3H), $2.26(\mathrm{~d}, J=5.9 \mathrm{~Hz}, 3 \mathrm{H}) ;{ }^{13} \mathrm{C}$ NMR $\left(101 \mathrm{MHz}, \mathrm{DMSO}-d_{6}\right)$ $\delta: 196.82,166.25,162.95,160.37,151.14,143.17,132.06$, $131.22,130.68,129.77,126.20,120.11,114.26,102.89$, 99.99, 26.98, 26.45, 22.04; HR-MS (ESI) calcd for $\mathrm{C}_{29} \mathrm{H}_{26} \mathrm{~N}_{5} \mathrm{O}_{3} \mathrm{~S}[\mathrm{M}+\mathrm{H}]^{+}$524.1756, found 524.1758.

$N$-(3-硝基苯乙烯基苯基酮)-2-(苯并咪唑-2-亚甲硫 基)-6-甲基嘧啶-4-胺(13w)：黄色粉末状固体，0.176 g, 收率 41.9\%. m.p. 170 $171{ }^{\circ} \mathrm{C}$; ${ }^{1} \mathrm{H}$ NMR $(400 \mathrm{MHz}$, DMSO- $\left.d_{6}\right) \delta: 10.65(\mathrm{~s}, 1 \mathrm{H}), 8.79(\mathrm{~s}, 1 \mathrm{H}), 8.41(\mathrm{~d}, J=7.8$ $\mathrm{Hz}, 1 \mathrm{H}), 8.29$ (dd, $J=8.2,1.5 \mathrm{~Hz}, 1 \mathrm{H}), 8.18$ (dd, $J=12.2$, $9.0 \mathrm{~Hz}, 3 \mathrm{H}), 7.90 \sim 7.67(\mathrm{~m}, 6 \mathrm{H}), 7.49(\mathrm{dd}, J=6.2,3.2 \mathrm{~Hz}$, $2 \mathrm{H}), 6.63(\mathrm{~s}, 1 \mathrm{H}), 4.96(\mathrm{~s}, 2 \mathrm{H}), 2.26(\mathrm{~s}, 3 \mathrm{H}) ;{ }^{13} \mathrm{C} \mathrm{NMR}$ $\left(101 \mathrm{MHz}, \mathrm{DMSO}-d_{6}\right) \delta: 187.58,166.65,160.38,151.43$, $148.90,143.94,141.23,137.20,135.42,132.07,131.25$, $130.84,130.51,126.19,125.22$, 125.05, 123.55, 119.87, 114.25, 103.12, 26.49, 22.62; HR-MS (ESI) calcd for $\mathrm{C}_{28} \mathrm{H}_{23} \mathrm{~N}_{6} \mathrm{O}_{3} \mathrm{~S}[\mathrm{M}+\mathrm{H}]^{+}$523.1552, found 523.1551.

$N$-(2-噻吩苯乙烯基苯基酮)-2-(苯并咪唑-2-亚甲硫 基)-6-甲基嘧啶-4-胺(13x)：黄色粉末状固体，0.082 g, 收率 33.9\%. m.p. 190 $191{ }^{\circ} \mathrm{C} ;{ }^{1} \mathrm{H}$ NMR (400 MHz, DMSO- $\left.d_{6}\right) \delta: 10.73(\mathrm{~s}, 1 \mathrm{H}), 8.02$ (d, $\left.J=8.8 \mathrm{~Hz}, 2 \mathrm{H}\right), 7.90$ $(\mathrm{d}, J=15.3 \mathrm{~Hz}, 1 \mathrm{H}), 7.81(\mathrm{~d}, J=5.0 \mathrm{~Hz}, 1 \mathrm{H}), 7.73$ (dd, $J=$ 6.0, 3.2 Hz, 3H), 7.64 (d, $J=8.8 \mathrm{~Hz}, 2 \mathrm{H}), 7.59$ (d, $J=15.3$ $\mathrm{Hz}, 1 \mathrm{H}), 7.49$ (dd, $J=6.1,3.2 \mathrm{~Hz}, 2 \mathrm{H}), 6.65$ (s, 1H), 4.97 $(\mathrm{s}, 2 \mathrm{H}), 2.27(\mathrm{~s}, 3 \mathrm{H}) ;{ }^{13} \mathrm{C}$ NMR (100 MHz, DMSO- $\left.d_{6}\right) \delta$ : $189.50,166.56,160.37,151.81,151.34,143.88,143.46$, $132.98,132.55,131.23,130.19,129.52,127.40,126.17$, $121.46,119.95,114.22,103.08,33.90,26.53,24.11,20.31$; HR-MS (ESI) calcd for $\mathrm{C}_{26} \mathrm{H}_{22} \mathrm{~N}_{5} \mathrm{OS}_{2}[\mathrm{M}+\mathrm{H}]^{+} 484.1266$, found 484.1266 .

$N$-(2-呋喃苯乙烯基苯基酮)-2-(苯并咪唑-2-亚甲硫 基)-6-甲基嘧啶-4-胺(13y)：黄色粉末状固体，0.155 g, 收率 66.2\%. m.p. 186 187 ${ }^{\circ} \mathrm{C} ;{ }^{1} \mathrm{H}$ NMR (400 MHz, DMSO- $\left.d_{6}\right) \delta: 10.68(\mathrm{~s}, 1 \mathrm{H}), 7.97(\mathrm{dd}, J=5.2,3.5 \mathrm{~Hz}, 3 \mathrm{H})$, $7.73(\mathrm{dd}, J=6.2,3.2 \mathrm{~Hz}, 2 \mathrm{H}), 7.63$ (d, $J=8.8 \mathrm{~Hz}, 2 \mathrm{H})$, $7.56(\mathrm{~s}, 2 \mathrm{H}), 7.50(\mathrm{dd}, \quad J=6.2,3.2 \mathrm{~Hz}, 2 \mathrm{H}), 7.14(\mathrm{~d}, J=$ $3.3 \mathrm{~Hz}, 1 \mathrm{H}), 6.72(\mathrm{dd}, J=3.4,1.8 \mathrm{~Hz}, 1 \mathrm{H}), 6.63(\mathrm{~s}, 1 \mathrm{H})$, 4.96 (s, 2H), 2.27 (s, 3H). ${ }^{13} \mathrm{C}$ NMR (100 MHz, DMSO- $d_{6}$ ) $\delta: 186.69,165.99,159.89,151.26,150.84,146.09,142.93$, $131.98,130.76,129.88,129.45,125.73,119.58,118.65$, $116.69,113.76,113.10,102.55,26.01,21.83$; HR-MS (ESI) calcd for $\mathrm{C}_{26} \mathrm{H}_{22} \mathrm{~N}_{5} \mathrm{O}_{2} \mathrm{~S}[\mathrm{M}+\mathrm{H}]^{+}$468.1494, found
468.1494 .

\subsection{4 抗肿瘤细胞毒活性实验}

初步篮选：取对数生长期的细胞，消化、计数，以 6000 个/孔的细胞密度接种至 96 孔板中, 每孔 $100 \mu \mathrm{L}$, 培养 $24 \mathrm{~h}$ 后，弃去培养基，加入提前用培养基稀释好的 样品, 每个孔 $200 \mu \mathrm{L}$ (浓度梯度为 50 和 $100 \mu \mathrm{g} / \mathrm{mL}$ ), 每 个浓度设 6 个复孔另设空白对照组及阴性对照组. 药物 作用 $72 \mathrm{~h}$ 后, 每孔加入 $5 \mu \mathrm{L} \mathrm{CCK}-8$ 进行染色, 继续培养 $4 \mathrm{~h}$ 后, 加入 $150 \mu \mathrm{L}$ 的二甲基亚砜, 振荡均匀, 酶标仪 $450 \mathrm{~nm}$ 处检测吸光度值，计算抑制率．进一步笁选：样 品抑制率大于 $50 \%$ 的样品进行半数抑制率 $\left(\mathrm{IC}_{50}\right)$ 实验. 即将待测样品以 $100,50,25,12.5,6.25,3.125,1.5625$, $0.78125 \mu \mathrm{g} / \mathrm{mL}$ 浓度加入 96 孔板中，实验方法同上，培 养 $72 \mathrm{~h}$ 后, 测 $450 \mathrm{~nm}$ 时的吸光度值, 通过 SPSS16.0 统 计软件计算得出 $\mathrm{IC}_{50}$ 值.

辅助材料(Supporting Information) 化合物 12a $\sim 120$ 和 13a 13y 的 ${ }^{1} \mathrm{H}$ NMR 和 ${ }^{13} \mathrm{C}$ NMR 谱图. 这些材料可 以免费从本刊网站(http://sioc-journal.cn/)上下载.

\section{References}

[1] For statistical information about cancer, see: U. S. Food and Drug Administration. http://www.fda.gov/About FDA/ReportsManualsForms/Reports/ucm276385.htm.

[2] Sangthong, S.; Krusong, K.; Ngamrojanavanich, N.; Vilaivan, T.; Puthong, S.; Chandchawan, S.; Muangsin, N. Bioorg. Med. Chem. Lett. 2011, 21, 4813.

[3] Cao, R. H.; Fan, W. X.; Guo, L.; Ma, Q.; Zhang, G. X.; Li, J. R.; Chen, X. M.; Ren, Z. H.; Qiu, L. Q. Eur. J. Med. Chem. 2013, 60, 135.

[4] Kamal, A.; Tamboli, J. R.; Nayak, V. L.; Adil, S. F.; Vishnuvardhan, M. V. P. S.; Ramakrishna, S. Bioorg. Med. Chem. Lett. 2013, 23 3208.

[5] Kamal, A.; Dastagiri, D.; Ramaiah, M. J.; Reddy, J. S.; Bharathi, E. V.; Reddy, M. K.; Sagar, M. V. P.; Reddy, T. L.; Pushpavalli, S. N. C. V. L.; Pal-Bhadra, M. Eur. J. Med. Chem. 2011, 46, 5817.

[6] Hamid, M. K. A. E.; Mihovilovic, M. D.; El-Nassan, H. B. Eur. J. Med. Chem. 2012, 57, 323.

[7] Kassab, A. E.; Gedawy, E. M. Eur. J. Med. Chem. 2013, 63, 224.

[8] Duschinsky, R.; Pleven, E.; Heidelberger, C. J. Am. Chem. Soc. 1957, 79, 4559.

[9] Pecchi, S.; Renhowe, P. A.; Taylor, C.; Kaufman, S.; Merritt, H.; Wiesmann, M.; Shoemaker, K. R.; Knapp, M.; Ornelas, E.; Hendrickson, T. F.; Fantl, W.; Voliva, C. F. Bioorg. Med. Chem. Lett. 2010, 20, 6895.

[10] Keche, A. P.; Hatnapure, G. D.; Tale, R. H.; Rodge, A. H.; Birajdar, S. S.; Kamble, V. M. Bioorg. Med. Chem. Lett. 2012, 22, 3445.

[11] Ni, L.; Meng, C. Q.; Sikorski, J. A. Expert. Opin. Ther. Pat. 2004, $14,1669$.

[12] Dimmock, J. R.; Elias, D. W.; Beazely, M. A. Kandepu, N. M. Curr. Med. Chem. 1999, 6, 1125.

[13] Domínguez, J. N.; León, C.; Rodrigues, J.; Gamboa, D. N.; Gut, J.; Rosenthal, P. J. J. Med. Chem. 2005, 48, 3654.

[14] Gacche, R.; Dhole, N. A.; Kamble, S. G.; Bandgar, B. P. J. Enzyme Inhib. Med. Chem. 2008, 23, 28.

[15] Sharma, V.; Kumar, P.; Pathak, D. J. Heterocycl. Chem. 2010, 47, 491.

[16] Shao, K. P.; Zhang, X. Yao.; Chen, P. J.; Xue, D. Q.; He, P.; Ma, L. 
Y.; Zheng, J. X.; Zhang, Q. R.; Liu, H. M. Bioorg. Med. Chem. Lett. 2014, 24, 3877.

[17] Chen, P. J.; Yang, A.; Gu, Y. F.; Zhang, X. S.; Shao, K. P.; Xue, D. Q.; He, P.; Jiang, T. F.; Zhang, Q. R.; Liu, H. M. Bioorg. Med.
Chem. Lett. 2014, 24, 2741.

[18] Tominaga, H.; Ishiyama, M.; Ohseto, F.; Sasamoto, K.; Hamamoto, T.; Suzuki, K.; Watanabe, M. Anal. Commun. 1999, 36, 47.

(Zhao, C.) 